\title{
SARS-CoV-2 neutralizing antibody structures inform therapeutic strategies
}

https://doi.org/10.1038/s41586-020-2852-1

Received: 30 August 2020

Accepted: 6 October 2020

Published online: 12 October 2020

Check for updates

\begin{abstract}
Christopher O. Barnes', Claudia A. Jette', Morgan E. Abernathy', Kim-Marie A. Dam1, Shannon R. Esswein', Harry B. Gristick', Andrey G. Malyutin ${ }^{2}$, Naima G. Sharaf ${ }^{3}$, Kathryn E. Huey-Tubman', Yu E. Lee', Davide F. Robbiani ${ }^{4,6}$, Michel C. Nussenzweig ${ }^{4,5}$, Anthony P. West Jr ${ }^{1}$ \& Pamela J. Bjorkman ${ }^{1 凶}$
\end{abstract}

The coronavirus disease 2019 (COVID-19) pandemic presents an urgent health crisis. Human neutralizing antibodies that target the host ACE2 receptor-binding domain (RBD) of the severe acute respiratory syndrome coronavirus-2 (SARS-CoV-2) spike protei ${ }^{1-5}$ show promise therapeutically and are being evaluated clinically ${ }^{6-8}$. Here, to identify the structural correlates of SARS-CoV-2 neutralization, we solved eight new structures of distinct COVID-19 human neutralizing antibodies ${ }^{5}$ in complex with the SARS-CoV-2 spike trimer or RBD. Structural comparisons allowed us to classify the antibodies into categories: (1) neutralizing antibodies encoded by the $\mathrm{VH} 3$-53 gene segment with short CDRH3 loops that block ACE2 and bind only to 'up' RBDs; (2) ACE2-blocking neutralizing antibodies that bind both up and 'down' RBDs and can contact adjacent RBDs; (3) neutralizing antibodies that bind outside the ACE2 site and recognize both up and down RBDs; and (4) previously described antibodies that do not block ACE2 and bind only to up RBDs 9 . Class 2 contained four neutralizing antibodies with epitopes that bridged RBDs, including a VH3-53 antibody that used a long CDRH3 with a hydrophobic tip to bridge between adjacent down RBDs, thereby locking the spike into a closed conformation. Epitope and paratope mapping revealed few interactions with host-derived $\mathrm{N}$-glycans and minor contributions of antibody somatic hypermutations to epitope contacts. Affinity measurements and mapping of naturally occurring and in vitro-selected spike mutants in 3D provided insight into the potential for SARS-CoV-2 to escape from antibodies elicited during infection or delivered therapeutically. These classifications and structural analyses provide rules for assigning current and future human RBD-targeting antibodies into classes, evaluating avidity effects and suggesting combinations for clinical use, and provide insight into immune responses against SARS-CoV-2.
Neutralizing antibodies (NAbs) against SARS-CoV-2 protect against infection in animal models ${ }^{1,3,4,10,11}$ and are being evaluated for prophylaxis and as therapeutic agents in humans $\mathrm{s}^{7,8}$. These antibodies target the SARS-CoV-2 spike (S) trimer ${ }^{3,5,10,12-17}$, a viral glycoprotein that mediates binding to the angiotensin-converting enzyme 2 (ACE2) receptor ${ }^{18,19}$. The S trimer comprises three copies of an S1 subunit that contains the RBD and three copies of S2, which includes the fusion peptide and transmembrane regions ${ }^{20,21}$. The RBDs of SARS-CoV-2 and other coronaviruses exhibit flexibility, such that they bind to ACE2 only when they are in an 'up' conformation, compared with the 'down' RBD conformation of the closed, prefusion $S$ trimer ${ }^{20-25}$.

Many human NAbs isolated from COVID-19-convalescent donors target the RBD, binding to distinct, sometimes non-overlapping, epitopes $^{3-5,10,12-14,17}$. A subset of these antibodies blocks viral entry by binding to the ACE2-binding site on the RBD ${ }^{6,11,13,15,26,27}$. A family of recurrent ACE2-blocking human NAbs is composed of heavy chains encoded by the $\mathrm{VH3}$-53 or $\mathrm{VH3}$-66 gene segment ${ }^{3,12,13,16,17,27-29}$, most of which are known or predicted ${ }^{15,26,28,30,31}$ to exhibit a common RBD binding mode that results from the use of germline-encoded residues within the complementarity-determining regions 1 and 2 (CDRH1 and $\mathrm{CDRH} 2$ ) and a CDRH 3 that is shorter than the average length ( 15 amino acids; IMGT $^{32}$ complementarity-determining region (CDR) definition) in human antibodies $^{33}$. Other SARS-CoV-2 RBD-binding antibodies are encoded by $\mathrm{VH} 3-3 \mathrm{O}^{5}$, and these have also been isolated from donors infected with SARS-CoV ${ }^{34}$, and antibodies with a variety of the other $\mathrm{VH}$ gene segments $\mathrm{s}^{3,5,10,12-17}$.

To classify commonalities and differences among RBD-binding human NAbs isolated from COVID-19-convalescent individuals ${ }^{5}$, we solved complexes of NAbs with stabilized (2P and 6P versions) ${ }^{35,36}$ soluble S trimer. Subsequently, we used high-resolution details of the

'Division of Biology and Biological Engineering, California Institute of Technology, Pasadena, CA, USA. ${ }^{2}$ Beckman Institute, California Institute of Technology, Pasadena, CA, USA. ${ }^{3}$ Division of Chemistry and Chemical Engineering, California Institute of Technology, Pasadena, CA, USA. ${ }^{4}$ Laboratory of Molecular Immunology, The Rockefeller University, New York, NY, USA. ${ }^{5} \mathrm{H} o w a r d$ Hughes Medical Institute, Chevy Chase, MD, USA. ${ }^{6}$ Present address: Institute for Research in Biomedicine, Università della Svizzera Italiana, Bellinzona, Switzerland.

凶e-mail: bjorkman@caltech.edu 
binding orientations of NAbs encoded by the $\mathrm{VH1}-2, \mathrm{VH1}-46, \mathrm{VH}-3 \mathrm{O}$, $\mathrm{VH} 3-53, \mathrm{VH} 4-34$ and $\mathrm{VH} 5$-51 gene segments to determine rules for binding by four distinct anti-RBD antibody classes (Supplementary Table 2). The NAbs chosen for structures are highly potent, achieving $90 \%$ neutralization in pseudotype virus assays at concentrations ranging from 22 to $140 \mathrm{ng} \mathrm{ml}^{-1}\left(\right.$ ref. $^{5}$ ), and thus our structural analyses and classifications directly relate to understanding mechanisms of neutralization and potency differences between human NAbs.

\section{Class 1 VH3-53 NAbs block ACE2 and bind to up RBDs}

We solved Fab and Fab-RBD crystal structures of C102 (Supplementary Table 1), which we compared to our previous ${ }^{26}$ cryo-electron microscopy (cryo-EM) structure of S trimer complexed with the related human NAb C105 (Extended Data Figs. 1, 2). Both $\mathrm{C} 102$ and C105 are VH3-53 NAbs with short (11 and 12 residues, respectively) CDRH3 loops (Extended Data Fig. 1g) that were isolated from the same donor $^{5}$. They share structural similarities with each other and with other VH3-53-encoded short CDRH3 human NAb structures solved as complexes with RBDs ${ }^{12,30,37,38}$ (Extended Data Fig. 2a). Notably, the C102-RBD structure resembled the analogous portion of the $\mathrm{C105-S}$ structure $^{26}$ (Extended Data Fig. 2a). These results establish that FabRBD structures can reproduce interactions with RBDs in the context of an S trimer; however, Fab-RBD structures do not reveal the state(s) of the antibody-bound RBD in the complex (up versus down) or the potential inter-protomer contacts by Fabs.

Because the C105 Fab bound to either two or three up RBDs on S with no observed interactions with down RBDs or with adjacent RBDs ${ }^{26}$ (Extended Data Fig. 1f), we used the higher-resolution C102 Fab-RBD structure to deduce a more accurate epitope and paratope than was possible using the C105-S cryo-EM structure with flexible up RBDs (Extended Data Fig. 1a-e). Buried surface area calculations showed that the $\mathrm{C} 102 \mathrm{CDRH} 3$ region had a relatively minor role in the paratope: of $1,045 \AA^{2}$ of buried surface area on the antibody ( $786 \AA^{2}$ on the heavy chain; $259 \AA^{2}$ on the light chain), CDRH3 accounted for only $254 \AA^{2}$ (Extended Data Fig. 2b). This contrasts with most antibodies in which $\mathrm{CDRH} 3$ contributes equally or more to the interface with antigen than the sum of CDRH1 and CDRH 2 contributions ${ }^{39}$. The epitopes on RBD for all available $V H 3$-53-encoded short $\mathrm{CDRH} 3$ human NAbs span the ACE2-binding site ${ }^{15,26,28,30,31}$ and show common RBD-binding interactions, represented by the $\mathrm{C} 102$ epitope (Extended Data Fig. 1b-e), which buried 1,017 $\AA^{2}$ on RBD (Extended Data Fig. 2b). The ACE2-blocking epitope for these NAbs is sterically occluded in the RBD down conformation (Fig. 1b, Extended Data Fig. 1f); therefore, class 1 NAbs can only bind to up RBDs, as observed in the C105-S structure ${ }^{26}$, and as previously discussed, IgGs in this class could crosslink adjacent RBDs within a single trimer to achieve tighter binding through avidity effects ${ }^{26}$.

\section{Class 2 NAbs recognize 'up' and 'down' RBDs}

In addition to the recurrent $\mathrm{VH3}-53$-encoded short CDRH3 NAb structures, a small subset of potently neutralizing $\mathrm{VH3}$-53-encoded antibodies use longer $\mathrm{CDRH} 3$ regions $^{5,12}$ (more than 15 residues, IMGT definition $^{32}$ ) (Extended Data Fig. 1g). A recent structure of a RBD complexed with a $\mathrm{VH} 3$-53-encoded long CDRH3 human NAb (COVA239) revealed a different RBD binding mode ${ }^{38}$, thus confirming predictions that binding with a $\mathrm{C102}$-like interaction requires a short $\mathrm{CDRH}^{26,30}$. To further determine molecular mechanisms for binding of $V H 3$-53-encoded long CDRH3 human NAbs, we solved a $3.2 \AA$ cryo-EM structure of $\mathrm{C} 144$ (encoded by the $V H 3-53$ and $V L 2-14$ gene segments; 25 -residue CDRH3) bound to an Strimer $^{36}$ (Extended Data Fig. 3). Despite the ability of ligand-free stabilized S trimers to adopt up RBD conformations ${ }^{36}$ and modelling suggesting the $\mathrm{C} 144$ binding site would be accessible on up RBDs (Fig. 1b), the C144-S structure revealed three $\mathrm{C} 144$ Fabs bound to a completely closed $\mathrm{S}$ with three down RBDs

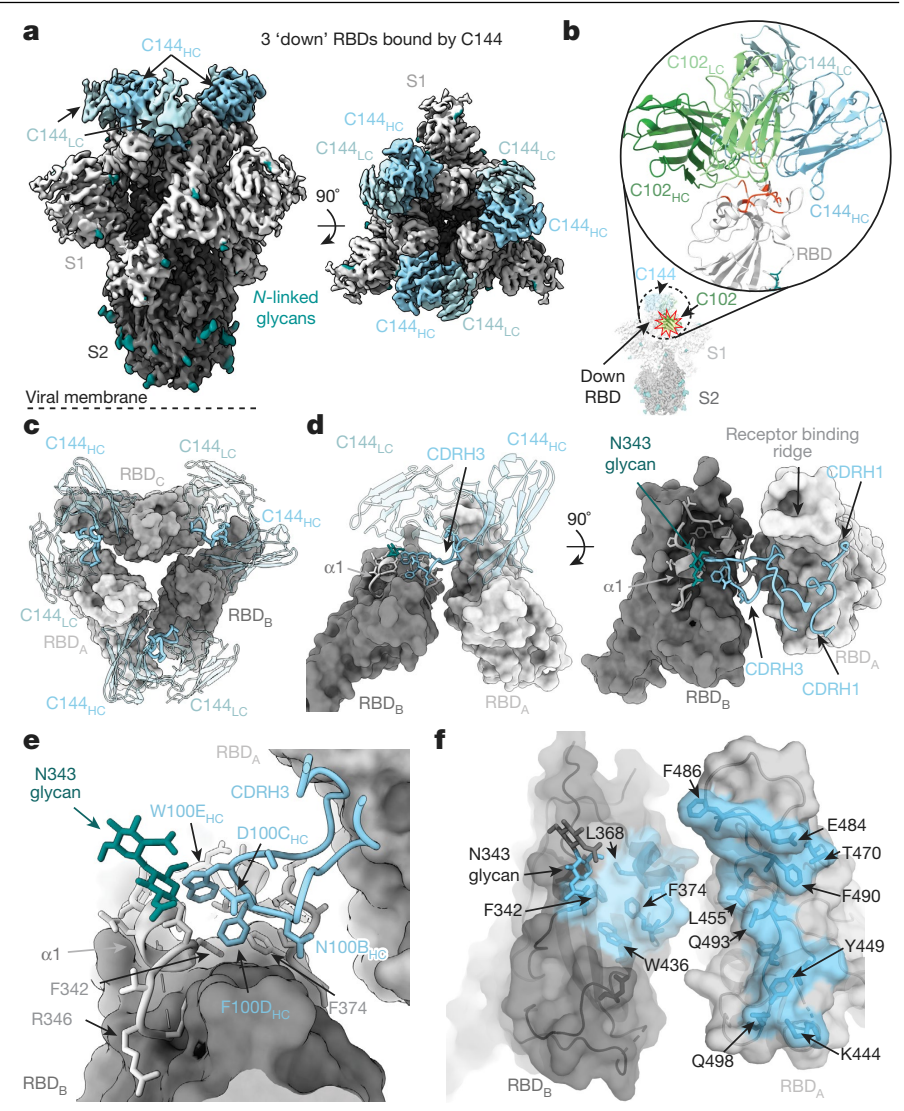

Fig. 1 | Cryo-EM structure of the C144-S complex illustrates a distinct VH353 NAb binding mode. a, 3.2 A cryo-EM density for the C144-S trimer complex revealing $\mathrm{C} 144$ binding to a closed (three down RBDs) spike conformation. LC, light chain; HC, heavy chain. b, Overlay of C102 Fab (from C102-RBD crystal structure) (Extended Data Fig. 1) and C144 Fab (from C144-S structure) aligned on a RBD monomer. RBD residues corresponding to the ACE2 epitope (orange-red cartoon) are shown on the same RBD for reference. C144 adopts a distinct conformation relative to the C102-like $V H 3$-53-encoded short CDRH3 NAb class, allowing binding to the down RBD conformation on trimeric spike, whereas C102-like NAbs can only bind to up RBDs. c, Quaternary epitope of $\mathrm{C} 144$ involving bridging between adjacent $\mathrm{RBDs}$ via the $\mathrm{CDRH} 3$ loop (illustrated as thicker ribbon). d, e, Close-up view of CDRH3-mediated contacts on adjacent protomer RBD (dark grey). $\mathrm{C} 144 \mathrm{CDRH} 3$ residues $\mathrm{F} 100_{\mathrm{D}}$ and $\mathrm{W} 100_{\mathrm{E}}$ are buried in a hydrophobic pocket comprising the RBD $\alpha 1$ helix, residue F $374_{\mathrm{RBD}}$ and the N343 ${ }_{\mathrm{RBD}}$ glycan.f, Surface representation of C144 epitope (light blue) across two adjacent RBDs. RBD epitope residues (defined as residues containing atom(s) within $4 \AA$ Å of a Fab atom) are labelled in black.

(Fig. 1a). The $\mathrm{C} 144$ binding mode differs from class 1 NAbs, the binding orientation of which is incompatible with down RBD conformations (Fig. 1b). In addition, the binding orientation observed for C144 differs from the binding described for COVA2-39, the RBD epitope of which is predicted to be accessible only on up RBDs ${ }^{38}$ owing to steric hinderances imposed on the light chain by the $N 343_{\mathrm{RBD}}$-associated glycan on the adjacent RBD (Extended Data Fig. 1h). Despite differences in orientation, the RBD epitopes of $\mathrm{C} 144, \mathrm{C} 102$ and COVA2-39 overlap with the ACE2-binding site, which suggests a neutralization mechanism that involves direct competition with ACE2 (Fig. 1b).

Despite overlapping with the ACE2-binding site on up RBDs, an interesting feature of $\mathrm{C} 144$ binding is that its long CDRH3 bridges between adjacent down RBDs to lock the spike glycoprotein into a closed, prefusion conformation, providing an additional neutralization mechanism in which S cannot open to engage ACE2 (Fig. 1c, d). The formation of the $\mathrm{C} 144$ quaternary epitope is driven by sandwiching $\mathrm{CDRH} 3$ residues $\mathrm{F} 100_{\mathrm{D}}$ and $\mathrm{W} 100_{\mathrm{E}}$ (in which subscripts denote numbering of the CDRH3 


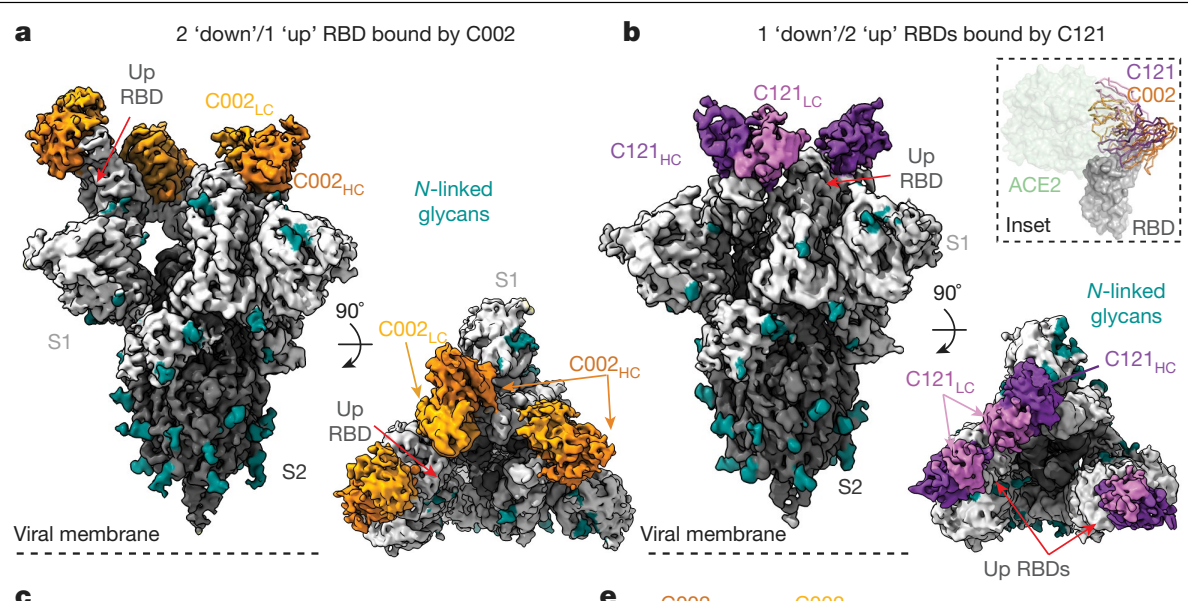

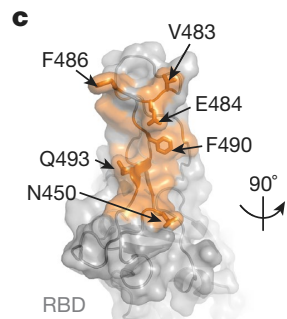
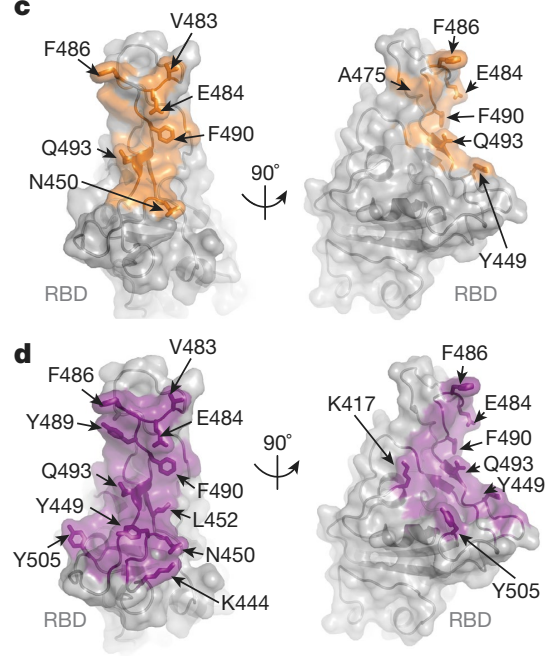

Fig. 2 | Cryo-EM structures of class $2 \mathrm{CO02}$ and $\mathrm{C121}$ NAbs show binding to up and down RBDs. a, b, Cryo-EM densities for C002-S (a; 3.4 $\AA$ ) and C121-S (b; $3.7 \AA$ A) complexes, revealing binding of $\mathrm{C} 002$ or $\mathrm{C} 121$ to both down and up RBDs. Inset, alignment of $\mathrm{COO} 2$ and $\mathrm{C} 121$ Fabs on the same RBD. ACE2 is represented as a green surface for reference. $\mathbf{c}, \mathbf{d}$, Surface representations of C002 epitope (orange, c) and C121 epitope (purple, d) on the RBD surface

loop) into a hydrophobic RBD cavity at the base of an $N$-linked glycan attached to $\mathrm{N} 343_{\mathrm{RBD}}$. The cavity comprises the RBD $\alpha 1$ helix (337-344), $\alpha 2$ helix (364-371), and hydrophobic residues ( $F 374_{\mathrm{RBD}}$ and $\left.\mathrm{W} 436_{\mathrm{RBD}}\right)$ at the edge of the RBD five-stranded $\beta$-sheet (Fig. 1e,f). In contrast to the CDRH3s of class $1 \mathrm{VH3}-53$-encoded short CDRH3 NAbs, the C144 CDRH3 contributed to most (approximately $60 \%$ ) of the paratope and buried $330 \AA^{2}$ of surface area on the adjacent RBD (Extended Data Fig. 2b), rationalizing observed escape at $\mathrm{L} 455_{\mathrm{RBD}}$ (Fig. If) in C144 selection experiments ${ }^{40}$. Despite adjacent $\mathrm{CDRH} 3$ hydrophobic residues $\left(\mathrm{F}_{100}\right.$ and $\mathrm{W}_{10 \mathrm{E}_{\mathrm{E}}}$ ) likely to be solvent-exposed before antigen binding, $\mathrm{C} 144$ IgG showed no evidence of non-specific binding in a polyreactivity assay (Extended Data Fig. 1i).

Given the unusual binding characteristics of $\mathrm{C} 144$, we investigated whether antibodies that showed similar S binding orientations in low-resolution negative-stain electron microscopy reconstructions ${ }^{5}$ use similar neutralization mechanisms. We characterized Fab-S cryo-EM structures (overall resolutions from 3.4 to $3.8 \AA$ ) of potent NAbs (C002, C104, C119 and C121) predicted to compete with ACE2 binding $^{5}$, which varied in their use of $V$ gene segments and CDRH3 lengths (Fig. 2, Extended Data Figs. 3, 4, Extended Data Table 1). Fab-Scryo-EM structures of these class 2 NAbs showed bound RBDs in both up or down conformations, consistent with observations of similar human NAbs from negative-stain electron microscopy ${ }^{5,12}$ and single-particle cryo-EM studies $^{10,34,41}$. By contrast, the C144-S structure showed Fabs bound

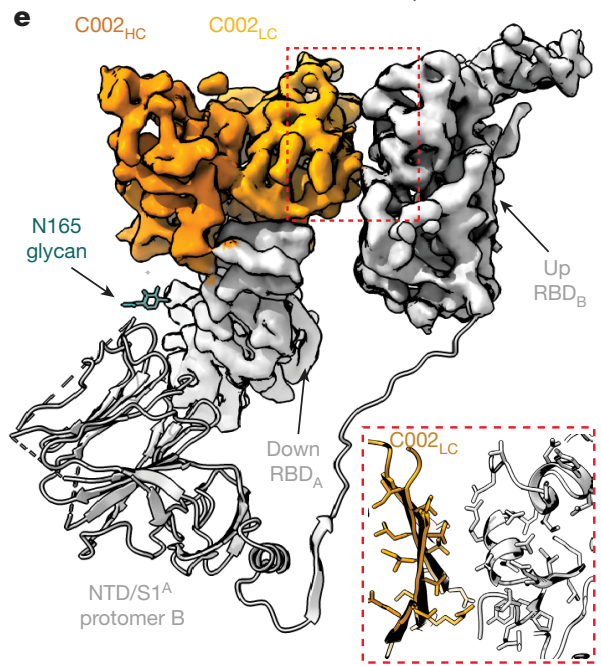

(grey). RBD epitope residues (defined as residues containing atom(s) within $4 \AA$ of a Fab atom) are labelled in black.e, $\mathrm{CO02}$ forms inter-protomer contacts via binding to an adjacent up RBD conformation on the surface of the trimer spike (also observed for class 2 C121-S, C119-S and C104-S structures) (Extended Data Fig. 5). Red box shows close-up of adjacent up RBD and C002 light-chain interface.

only to down RBDs (Fig. 1), which suggests that C144 binding requires recognition of the closed S trimer, or that $\mathrm{C} 144 \mathrm{Fab}$ (s) initially bound to up RBD(s) could trap the closed (three RBDs down) S conformation through CDRH3-mediated interactions between adjacent RBDs.

To understand commonalities of class 2 RBD epitopes better, we further analysed two additional potent human NAbs, C002 (encoded by $V H 3-3 O$ and $V K 1$-39 gene segments; 17-residue CDRH3, half-maximal inhibitory concentration $\left.\left(\mathrm{IC}_{50}\right)=8.0 \mathrm{ng} \mathrm{ml}^{-1}\right)^{5}$ and $\mathrm{C} 121$ (encoded by VH1-2 and VL2-23 gene segments; 23-residue CDRH3, $\left.\mathrm{IC}_{50}=6.7 \mathrm{ng} \mathrm{ml}^{-1}\right)^{5}$, for which cryo-EM Fab-S structures were solved to $3.4 \AA$ and $3.6 \AA$, respectively (Fig. 2a, b), using crystal structures of unbound $\mathrm{C002}$ and C121 Fabs for fitting (Supplementary Table 1). The C002 and C121 RBD epitopes are focused on the receptor-binding ridge, overlapping with polar and hydrophobic residues along the flat face of the RBD responsible for ACE2 interactions (Fig. 2c-e). Similar to C144, NAbs C002 and C121 buried most of their RBD epitopes against heavy-chain CDR loops, with light-chain CDR loops engaging the receptor-binding ridge (Fig. 3). Notably, Fab-S structures of C002, C121, C119 and C104 revealed a quaternary epitope involving an adjacent RBD (Extended Data Figs. 3, 4, 5a-c), albeit distinct from the quaternary binding of $\mathrm{C} 144$ (Fig. 1c-e). The C002/C121/C119/C104 type of secondary interaction was only observed when a Fab was bound to a down RBD and adjacent to an up RBD. The extent of secondary interactions varied depending on the antibody pose (Extended Data Fig. 5a-c). Bridging interactions 

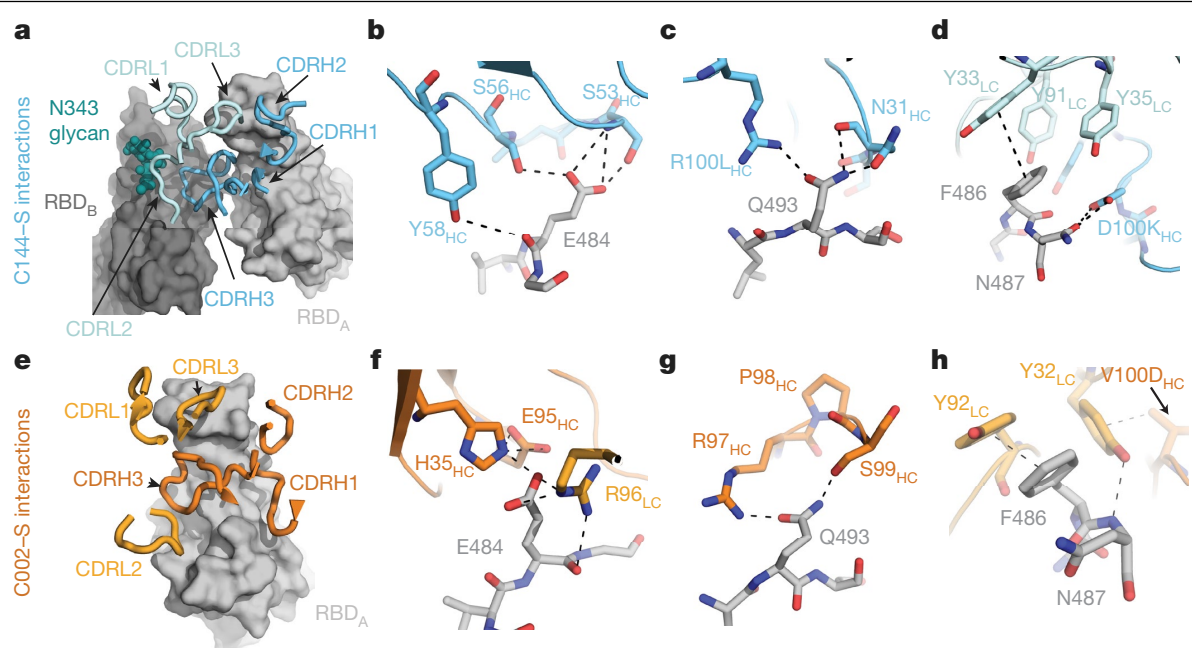

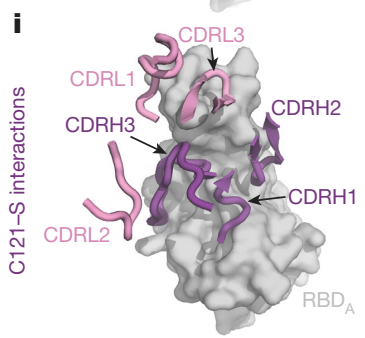

j

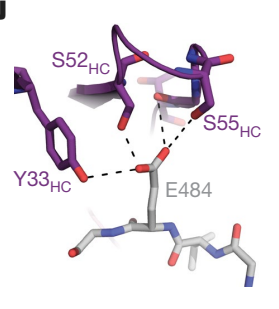

$\mathbf{k}$

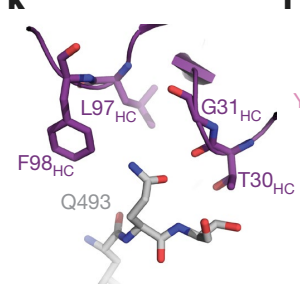

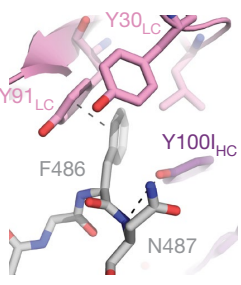

Fig. 3 | Details of common RBD interactions among class 2 human NAbs. a-l, Conserved interactions between the RBD and CDRs of class 2 NAbs as observed for C144 (HC, cyan; LC, sky blue) (a-d), C002 (HC, dark orange; LC, light orange) (e-h), and C121 (HC, purple; LC, pink) (i-l). Primary and secondary epitopes on adjacent down RBDs are shown for C144. Secondary epitopes for
C002 and C121, which require adjacent up RBDs, are shown in Extended Data Fig. 5. RBDs are grey; potential hydrogen bonds and $\pi-\pi$ stacking interactions (d, $\mathrm{Y}_{33_{\mathrm{LC}}}$ and $\mathrm{F} 486_{\mathrm{RBD}} ; \mathbf{h}, \mathrm{Y} 92_{\mathrm{LC}}$ and $\mathrm{F} 486_{\mathrm{RBD}} ; \mathbf{1}, \mathrm{Y} 91_{\mathrm{LC}}$ and $\mathrm{F} 486_{\mathrm{RBD}}$ ) are indicated by dashed lines. between adjacent up and down RBDs would not allow the two Fabs of a single IgG to bind simultaneously to an S trimer. However, this class of antibodies could support bivalent interactions between two adjacent down RBDs (Extended Data Fig. 5h, Extended Data Table 1).

Characterization of the highest resolution interface (CO02-Sstructure) showed C002 light-chain framework regions 1 and 2 (FWR1 and FWR2) interfaced with the RBD residues comprising the five-stranded $\beta$-sheet and $\alpha$-helix that spans residues $440-444$ (Fig. 2e), which is typically located near the three-fold axis of a closed Strimer. In addition to contacting neighbouring RBDs, inter-protomer engagement with the $\mathrm{N} 165_{\mathrm{NTD}}$-associated glycan in the N-terminal domain (NTD) was observed for the class $2 \mathrm{NAb}$ $\mathrm{BD} 23^{13}$. If fully processed, the $\mathrm{N} 165_{\mathrm{NTD}}$ glycan could adopt a conformation that would allow interactions with the heavy-chain FWR3 and CDRH1 (Fig. 2e). However, in the structures reported here, we did not observe $\mathrm{N} 165_{\mathrm{NTD}}$ glycan density beyond the initial $\mathrm{N}$-acetylglucosamine.

Given differences in class 2 human NAb V gene segments, CDRH3 lengths and antibody poses, we investigated sequence features that drive conserved interactions. Sequence differences between SARS-CoV-2 and SARS-CoV RBD, including at positions $486_{\mathrm{RBD}}$ and $493_{\mathrm{RBD}}$ ( $F$ and $Q$, respectively, in SARS-CoV-2), in the ACE2 receptor-binding motif allowed more favourable ACE2 binding to the SARS-CoV-2 RBD ${ }^{42}$. Analysis of interactions by $\mathrm{C} 144, \mathrm{C} 002$ and $\mathrm{C} 121$ revealed common interactions with these residues and also for $\mathrm{E} 484_{\mathrm{RBD}}$ by both antibody heavy-chain and light-chain residues (Fig. 3). In particular, class $2 \mathrm{NAb}$ interactions with $\mathrm{F} 486_{\mathrm{RBD}}$ mimicked $\mathrm{ACE} 2$ interactions, in that $\mathrm{F} 486_{\mathrm{RBD}}$ buries into a hydrophobic pocket typically involving CDRL1 or CDRL3 tyrosine residues ${ }^{43}$ (Fig. 3d, h, l). Mimicking of the ACE2 F486 binding pocket by SARS-CoV-2 human NAbs was observed across different light-chain V gene segments (Extended Data Table 1), which suggests that there is no restriction in light-chain $V$ gene segment usage for class 2 NAbs. Notably, a germline-encoded feature described for $\mathrm{VH3}$ 53-encoded short CDRH3 class 1 NAbs, the CDRH2 SXXS motif, is also found in other class 2 NAbs (for example, C121 and C119) despite different VH gene segment usage. Similar to VH3-53 NAbs C144 and COVA2-39, the C121 CDRH2SXXS motif forms a potential hydrogen-bond network with $\mathrm{E} 84_{\mathrm{RBD}}$ (Fig. 3b,j).

Overall, these results suggest a convergent mode of recognition by germline-encoded residues across diverse $\mathrm{VH} / \mathrm{VL}$ gene segments for SARS-CoV-2, which may contribute to low levels of somatic hypermutation observed for these human NAbs (Extended Data Fig. 4i-n, Extended Data Table 1).

\section{Class 3 NAbs bind outside the ACE2-binding site}

$\mathrm{C} 135$ is a potent NAb that showed distinct binding properties from class 1, 2 and 4 NAbs, the latter of which bind a highly conserved buried epitope that is only accessible in up RBD conformations (Extended Data Table1). To evaluate the mechanism of C135-mediated neutralization of SARS-CoV-2, we solved the cryo-EM structure of a C135-S complex to $3.5 \AA$ (Fig. 4a, Extended Data Fig. 6), using an unbound $\mathrm{C} 135$ crystal structure for fitting (Supplementary Table 1). The structure revealed three $\mathrm{C} 135$ Fabs bound to an S trimer with two down and one up RBD, although the C135-bound up RBD conformation was weakly resolved and therefore not modelled. C135 recognizes a similar glycopeptidic epitope to the cross-reactive SARS-CoV NAb S30 $9^{34}$, focusing on a region of the RBD near the N343 glycan and non-overlapping with the ACE2-binding site (Fig. 4b, Extended Data Fig. 6c, d). Despite differences in binding orientations between $\mathrm{C} 135$ and S309, targeting of the RBD epitope was mainly VH-mediated (the surface area buried by RBD on the $\mathrm{C} 135$ heavy chain represented approximately $480 \AA^{2}$ of the $700 \AA^{2}$ total buried surface area) and included interactions with the core fucose moiety of the N343 $3_{\mathrm{RBD}}$ glycan. The smaller C135 footprint relative to 3309 (approximately $700 \AA^{2}$ versus 1,150 $\AA^{2}$ buried surface area, respectively) (Extended Data Fig. 6c, d) focused on interactions with $\mathrm{R} 346_{\mathrm{RBD}}$ and $\mathrm{N} 440_{\mathrm{RBD}}$, which are engaged 


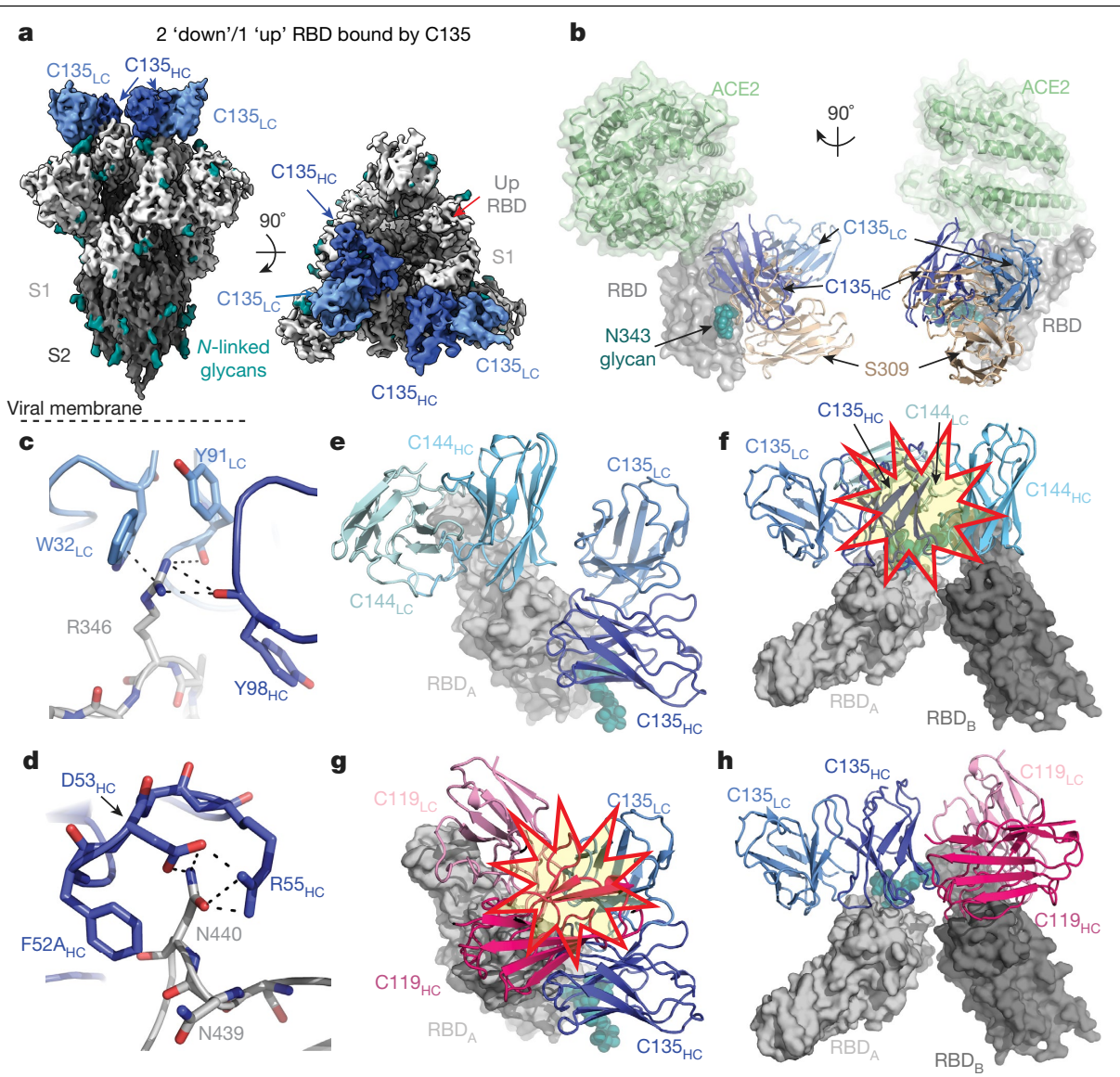

Fig. 4 | Cryo-EM structure of $S$ complexed with the class 3 (non-ACE2 blocking) human NAb C135. a, 3.5 Å cryo-EM density of C135-S complex. b, Composite model of C135-RBD (blue and grey, respectively) overlaid with the SARS-CoV-2 NAb S309 (sand; PDB code 6WPS) and soluble ACE2 (green; PDB code 6MOJ). The model was generated by aligning on $188 \mathrm{RBD} C \alpha$ atoms. c, d, C135 CDRH (dark blue) and CDRL (light blue) interactions with R346 ${ }_{\mathrm{RBD}}$ (c) and $\mathrm{N} 440_{\mathrm{RBD}}(\mathbf{d})$. Potential $\pi-\pi$ stacking interactions $(\mathbf{c})$ and hydrogen bonds (c, d) are illustrated by dashed black lines. e,f, Model of RBD interactions of

by residues from heavy-chain and light-chain CDRs (Fig. 4c, d) and are not conserved between SARS-CoV-2 and SARS-CoVRBDs, rationalizing the lack of SARS-CoV cross-reactivity observed for $\mathrm{C}^{135^{5}}$.

The discovery of class $3 \mathrm{NAbs}$ such as $\mathrm{C} 135$ and $\mathrm{S} 309$ that were raised during SARS-CoV-2 or SARS-CoV natural infections, respectively, and bind outside of the ACE2-binding site, provides the potential for additive neutralization effects when combined with NAbs that block ACE2, while also limiting viral escape ${ }^{1,40}$. A pair of antibodies in human clinical trials that includes REGN10987 ${ }^{8}$, a human NAb that binds distal to the ACE2-binding site, prevented SARS-CoV-2 viral escape in vitro, but did not show synergistic neutralization ${ }^{6}$. Comparison of $\mathrm{C} 135$ and REGN10987 interactions with S showed similarities in epitopes (interactions focused on R346 ${ }_{\mathrm{RBD}}$ and $\mathrm{N} 440_{\mathrm{RBD}}$ ) (Extended Data Fig. 7c, f). However, REGN10987 binding would sterically hinder ACE2 interactions, whereas $\mathrm{C} 135$ binding does not (Fig. 4b, Extended Data Fig. 6b). Notably, a structure of S complexed with C110 (encoded by the VH5-51 and VK1-5 gene segments), isolated from the same donor as the $\mathrm{C} 102$ and $\mathrm{C} 105$ (class 1) and $\mathrm{C} 119$ and $\mathrm{C} 121$ (class 2) NAbs ${ }^{5}$, showed a binding pose that resembled that of REGN10987 (Extended Data Fig. 6b, e, f). The C110 epitope showed similarities with both class 3 and class 2 NAbs, binding distal to the ACE2-binding motif, but like REGN10987, could potentially sterically interfere with ACE2 (Extended Data Fig. 7). For each of these class 3 NAbs, the Fab binding pose suggests that inter-protomer crosslinking by a single IgG is not possible (Extended Data Table 1).
NAbs C135 (class 3) and C144 (class 2), demonstrating that both Fabs can bind simultaneously to a single monomeric RBD (e), but would clash if bound to adjacent down RDBs on S trimer (f). Steric clashes indicated by a red and yellow star in f. g, h, Model of RBD interaction of NAbs C135 (class 3) and C119 (class 2) demonstrating that both Fabs cannot bind simultaneously to a single monomeric RBD (g), but do not clash if bound to adjacent down RDBs on S trimer (h). Steric clashes indicated by a red and yellow star in $\mathbf{g}$.
Class 3 human NAbs add to the anti-SARS-CoV-2 antibody repertoire and could probably be effectively used in therapeutic combinations with class 1 or 2 NAbs. However, when using structures to predict whether NAbs have overlapping epitopes, it is sometimes not sufficient to only examine Fab-RBD structures or even static images of the $S$ trimer because of the dynamic nature of the spike. Thus, what might appear to be non-overlapping epitopes on an isolated RBD could overlap in some (Fig. 4e,f), but not all (Extended Data Fig. 7), scenarios on a spike trimer, complicating interpretation of competition experiments using monomeric RBDs and S trimers. The opposite can also be true; that is, two Fabs predicted to be accommodated on a trimer could clash on an RBD monomer (Fig. 4g, h). Finally, adjacent monomers in different orientations could accommodate different antibodies that target overlapping sites (Extended Data Fig. 7).

\section{RBD substitutions affect NAb binding}

Vesicular stomatitis virus (VSV) reporter viruses pseudotyped with the SARS-CoV-2S protein can escape by mutation(s) from the C121, C135 or $\mathrm{C} 144 \mathrm{NAbs}^{40}$ that we used for structural studies. RBD mutations that were selected in response to antibody pressure correlated with the epitopes mapped from the structures of their Fabs complexed with the S trimer (Figs. 1, 2, 4). 
To further assess the effects of these mutations and other RBD substitutions, we assayed NAbs for which we obtained structural information (eight from this study; C105-S complex previously described ${ }^{26}$ ) for binding to mutated RBD proteins. The RBD mutants included two that induced escape from the class $3 \mathrm{hNAb}$ C135 (R346S and N440K) ${ }^{40}$ (Fig. 4c, d), one found in circulating isolates ${ }^{44}$ that conferred partial resistance to $\mathrm{C} 135$ (N439K) ${ }^{40}$ (Fig. 4d), a circulating variant (A475V) that conferred resistance to class 1 and $2 \mathrm{VH} 3-53 \mathrm{NAbs}^{44}$, two that induced escape from $\mathrm{C} 121$ or $\mathrm{C} 144$ (E484K and Q493R) ${ }^{40}$ (Fig. 3), and a circulating variant that conferred partial resistance to $\mathrm{C} 121(\mathrm{~V} 483 \mathrm{~A})^{40}$. Kinetic and equilibrium constants for the original and mutant RBDs were derived from surface plasmon resonance (SPR) binding assays in which RBDs were injected over immobilized IgGs. Loss of binding affinity was consistent with RBD mutations that conferred escape (Extended Data Fig. 8). Comparing effects of point mutations between NAb classes showed that point mutations leading to a loss of binding for NAbs within one class did not affect NAbs in a different class, indicating that antibody pressure that leads to escape from one NAb class would be unlikely to affect a different class. These results suggest a therapeutic strategy involving human NAbs of different classes for monoclonal NAb treatment of individuals infected with SARS-CoV-2.

\section{Conclusions}

Here we report structural, biophysical and bioinformatics analyses of SARS-CoV-2 NAbs (Extended Data Fig. 9), providing information for interpreting correlates of protection for clinical use. The structures reveal a wealth of unexpected interactions of NAbs with the spike protein, including five antibodies that reach between adjacent RBDs on the protomers of a single trimer. A notable example of bridging between spike protomers involved the human $\mathrm{C} 144 \mathrm{NAb}$ that uses a long $\mathrm{CDRH} 3$ with a hydrophobic tip to reach across to an adjacent RBD, resulting in all three RBDs on the spike trimer being locked into a closed conformation. This example, and four other NAbs that contact adjacent RBDs, demonstrates that crystal structures of Fab-monomeric RBD complexes, although informative for defining primary epitopes on one RBD, do not reveal how antibodies recognize the flexible up or down RBD conformations on the spike trimer that are targeted for neutralization on a virus. Indeed, our cryo-EM structures of Fab-spike trimer complexes showed all possible up and down combinations of recognized RBDs, with structures showing either three or two Fabs bound per trimer. By analysing approach angles of Fabs bound to RBDs on spike trimers, we predicted whether an IgG can bind to a single spike trimer to gain potency through avidity, which would also render the antibody more resistant to spike mutations. In addition, structural information allowed us to assess RBD mutants that arose in circulating viral isolates and/or were obtained by in vitro selection. Together, this study provides a blueprint for the design of antibody cocktails for therapeutic agents and potential spike-based immunogens for vaccines.

\section{Online content}

Any methods, additional references, Nature Research reporting summaries, source data, extended data, supplementary information, acknowledgements, peer review information; details of author contributions and competing interests; and statements of data and code availability are available at https://doi.org/10.1038/s41586-020-2852-1.

1. Baum, A. et al. REGN-COV2 antibody cocktail prevents and treats SARS-CoV-2 infection in rhesus macaques and hamsters. Science https://doi.org/10.1126/science.abe2402 (2020).

2. Baum, A. et al. Antibody cocktail to SARS-CoV-2 spike protein prevents rapid mutational escape seen with individual antibodies. Science 369, 1014-1018 (2020).

3. Rogers, T. F. et al. Isolation of potent SARS-CoV-2 neutralizing antibodies and protection from disease in a small animal model. Science 369, 956-963 (2020).

4. Zost, S. J. et al. Potently neutralizing and protective human antibodies against SARS-CoV-2. Nature 584, 443-449 (2020).

5. Robbiani, D. F. et al. Convergent antibody responses to SARS-CoV-2 in convalescent individuals. Nature 584, 437-442 (2020).
6. Hansen, J. et al. Studies in humanized mice and convalescent humans yield a SARS-CoV-2 antibody cocktail. Science 369, 1010-1014 (2020).

7. US National Library of Medicine. A study of LY3819253 (LY-CoV555) in preventing SARS-CoV-2 infection and COVID-19 in nursing home residents and staff (BLAZE-2). ClinicalTrials.gov https://clinicaltrials.gov/ct2/show/NCTO4497987 (2020).

8. NIH. Clinical trials of monoclonal antibodies to prevent COVID-19 now enrolling. https:// www.nih.gov/news-events/news-releases/clinical-trials-monoclonal-antibodies-preventcovid-19-now-enrolling (2020).

9. Yuan, M. et al. A highly conserved cryptic epitope in the receptor binding domains of SARS-CoV-2 and SARS-CoV. Science 368, 630-633 (2020).

10. Liu, L. et al. Potent neutralizing antibodies against multiple epitopes on SARS-CoV-2 spike. Nature 584, 450-456 (2020).

11. Kreye, J. et al. A SARS-CoV-2 neutralizing antibody protects from lung pathology in a COVID-19 hamster model. Preprint at https://doi.org/10.1101/2020.08.15.252320 (2020).

12. Brouwer, P. J. M. et al. Potent neutralizing antibodies from COVID-19 patients define multiple targets of vulnerability. Science 369, 643-650 (2020).

13. Cao, Y. et al. Potent neutralizing antibodies against SARS-CoV-2 identified by highthroughput single-cell sequencing of convalescent patients' B cells. Cell 182, 73-84 (2020).

14. Kreer, C. et al. Longitudinal isolation of potent near-germline SARS-CoV-2-neutralizing antibodies from COVID-19 patients. Cell 182, 843-854 (2020).

15. Shi, R. et al. A human neutralizing antibody targets the receptor-binding site of SARS-CoV-2. Nature 584, 120-124 (2020).

16. Zost, S. J. et al. Rapid isolation and profiling of a diverse panel of human monoclonal antibodies targeting the SARS-CoV-2 spike protein. Nat. Med. 26, 1422-1427 (2020).

17. Seydoux, E. et al. Analysis of a SARS-CoV-2-infected individual reveals development of potent neutralizing antibodies with limited somatic mutation. Immunity 53, 98-105 (2020).

18. Hoffmann, M. et al. SARS-CoV-2 cell entry depends on ACE2 and TMPRSS2 and is blocked by a clinically proven protease inhibitor. Cell 181, 271-280 (2020).

19. Wang, Q. et al. Structural and functional basis of SARS-CoV-2 entry by using human ACE2. Cell 181, 894-904 (2020).

20. Walls, A.C. et al. Structure, function, and antigenicity of the SARS-CoV-2 spike glycoprotein. Cell 181, 281-292 (2020).

21. Wrapp, D. et al. Cryo-EM structure of the 2019-nCoV spike in the prefusion conformation. Science 367, 1260-1263 (2020).

22. Walls, A. C. et al. Cryo-electron microscopy structure of a coronavirus spike glycoprotein trimer. Nature 531, 114-117 (2016).

23. Yuan, Y. et al. Cryo-EM structures of MERS-CoV and SARS-CoV spike glycoproteins reveal the dynamic receptor binding domains. Nat. Commun. 8, 15092 (2017).

24. Kirchdoerfer, R. N. et al. Pre-fusion structure of a human coronavirus spike protein. Nature 531, 118-121 (2016)

25. Li, Z. et al. The human coronavirus HCoV-229E S-protein structure and receptor binding. elife 8 , e51230 (2019).

26. Barnes, C.O. et al. Structures of human antibodies bound to SARS-CoV-2 spike reveal common epitopes and recurrent features of antibodies. Cell 182, 828-842 (2020).

27. Ju, B. et al. Human neutralizing antibodies elicited by SARS-CoV-2 infection. Nature $\mathbf{5 8 4}$ $115-119(2020)$

28. Wu, Y. et al. A noncompeting pair of human neutralizing antibodies block COVID-19 virus binding to its receptor ACE2. Science 368, 1274-1278 (2020).

29. Chi, X. et al. A neutralizing human antibody binds to the $\mathrm{N}$-terminal domain of the Spike protein of SARS-CoV-2. Science 369, 650-655 (2020).

30. Yuan, M. et al. Structural basis of a shared antibody response to SARS-CoV-2. Science 369, 1119-1123 (2020).

31. Hurlburt, N. K. et al. Structural basis for potent neutralization of SARS-CoV-2 and role of antibody affinity maturation. Preprint at https://doi.org/10.1101/2020.06.12.148692 (2020).

32. Lefranc, M. P. et al. IMGT ${ }^{\circledR}$, the international ImMunoGeneTics information system ${ }^{\circledR} 25$ years on. Nucleic Acids Res. 43, D413-D422 (2015).

33. Briney, B., Inderbitzin, A., Joyce, C. \& Burton, D. R. Commonality despite exceptional diversity in the baseline human antibody repertoire. Nature 566, 393-397 (2019).

34. Pinto, D. et al. Cross-neutralization of SARS-CoV-2 by a human monoclonal SARS-CoV antibody. Nature 583, 290-295 (2020).

35. Pallesen, J. et al. Immunogenicity and structures of a rationally designed prefusion MERS-CoV spike antigen. Proc. Natl Acad. Sci. USA 114, E7348-E7357 (2017).

36. Hsieh, C. L. et al. Structure-based design of prefusion-stabilized SARS-CoV-2 spikes. Science 369, 1501-155 (2020).

37. Wu, F. et al. Neutralizing antibody responses to SARS-CoV-2 in a COVID-19 recovered patient cohort and their implications. Preprint at https://doi.org/10.1101/2020.03.30.20047365 (2020).

38. Wu, N. C. et al. An alternative binding mode of IGHV3-53 antibodies to the SARS-CoV-2 receptor binding domain. Cell Rep. https://doi.org/10.1016/j.celrep.2020.108274 (2020).

39. Marillet, S., Lefranc, M. P., Boudinot, P. \& Cazals, F. Novel structural parameters of Ig-Ag complexes yield a quantitative description of interaction specificity and binding affinity. Front. Immunol. 8, 34 (2017).

40. Weisblum, Y. et al. Escape from neutralizing antibodies by SARS-CoV-2 spike protein variants. Preprint at https://doi.org/10.1101/2020.07.21.214759 (2020).

41. Wang, B. et al. Bivalent binding of a fully human IgG to the SARS-CoV-2 spike proteins reveals mechanisms of potent neutralization. Preprint at https://doi.org/10.1101/2020.07.14 203414 (2020).

42. Shang, J. et al. Structural basis of receptor recognition by SARS-CoV-2. Nature 581, 221-224 (2020).

43. Yan, R. et al. Structural basis for the recognition of SARS-CoV-2 by full-length human ACE2. Science 367, 1444-1448 (2020).

44. Li, Q. et al. The impact of mutations in SARS-CoV-2 spike on viral infectivity and antigenicity. Cell 182, 1284-1294.e9 (2020).

Publisher's note Springer Nature remains neutral with regard to jurisdictional claims in published maps and institutional affiliations.

(c) The Author(s), under exclusive licence to Springer Nature Limited 2020 


\section{Methods}

No statistical methods were used to predetermine sample size. The experiments were not randomized, and investigators were not blinded to allocation during experiments and outcome assessment.

\section{Cell lines}

Expi293F cells (GIBCO) for protein expression were maintained at $37^{\circ} \mathrm{C}$ and $8 \% \mathrm{CO}_{2}$ in Expi293 Expression medium (GIBCO), transfected using Expi293 Expression System Kit (GIBCO) and maintained under shaking at $130 \mathrm{rpm}$. Cell lines were not specifically authenticated, but lines tested negative for contamination with mycoplasma.

\section{Protein expression}

Expression and purification of SARS-CoV-2 ectodomains were conducted as previously described ${ }^{26}$. In brief, constructs encoded the SARS-CoV-2 S ectodomain (residues 16-1206 of the early SARS-CoV-2 GenBank MN985325.1 sequence isolate with $2 \mathrm{P}^{35}$ or $6 \mathrm{P}^{36}$ stabilizing mutations, a mutated furin cleavage site between S1 and S2, a C-terminal TEV site, foldon trimerization motif, octa-His tag, and AviTag) were used to express soluble SARS-CoV-2 S ectodomains. Constructs encoding the SARS-CoV-2 RBD from GenBank MN985325.1 (residues 331-524 with C-terminal octa-His tag and AviTag) and mutant RBDs were made as described ${ }^{26}$,SARS-CoV-2 2PS, 6PS, and RBD proteins were purified from the supernatants of transiently transfected Expi293F cells (Gibco) by nickel affinity and size-exclusion chromatography ${ }^{26}$. Peak fractions were identified by SDS-PAGE, and fractions corresponding to $S$ trimers or monomeric RBDs were pooled and stored at $4{ }^{\circ} \mathrm{C}$. Fabs and IgGs were expressed, purified, and stored as previously described ${ }^{45,46}$.

\section{X-ray crystallography}

Crystallization trials were carried out at room temperature using the sitting drop vapour diffusion method by mixing equal volumes of a Fab or Fab-RBD complex and reservoir using a TTP LabTech Mosquito robot and commercially available screens (Hampton Research). Crystals were obtained in $0.2 \mathrm{M}$ ammonium sulfate, 20\% (w/v) PEG 3350 (C102 Fab), $0.2 \mathrm{M}$ sodium citrate tribasic, $20 \%$ (w/v) PEG 3350 (C102-RBD), $0.2 \mathrm{M}$ lithium sulfate monohydrate, 20\% (w/v) PEG 3350 (C002 Fab), $0.04 \mathrm{M}$ potassium phosphate, $16 \%(\mathrm{w} / \mathrm{v})$ PEG $8000,20 \%(\mathrm{v} / \mathrm{v})$ glycerol (C135 Fab), 0.2 M ammonium citrate pH 5.1, 20\% PEG 3350 (C121 Fab), or 0.2 M sodium tartrate dibasic dihydrate $\mathrm{pH} 7.3,20 \%$ (w/v) PEG 3350 (C110 Fab). A C135 Fab crystal was directly looped and cryopreserved in liquid nitrogen. Other crystals were quickly cryoprotected in a mixture of well solution with $20 \%$ glycerol and then cryopreserved in liquid nitrogen.

$\mathrm{X}$-ray diffraction data were collected for Fabs and the Fab-RBD complex at the Stanford Synchrotron Radiation Lightsource (SSRL) beamline 12-1 on a Eiger X $16 \mathrm{M}$ pixel detector (Dectris) at a wavelength of $1.0 \AA$. Data from single crystals of $\mathrm{C} 121 \mathrm{Fab}$ and $\mathrm{C} 110 \mathrm{Fab}$ were indexed and integrated in $\mathrm{XDS}^{47}$ and merged using AIMLESS v.0.7.4 in CCP4 ${ }^{48}$ v.7.0.6 (Supplementary Table1). Data from single crystals of C102 Fab, $\mathrm{C} 135 \mathrm{Fab}$ and $\mathrm{C002} \mathrm{Fab}$ were indexed and integrated using $\mathrm{XDS}^{47}$ and merged in Phenix ${ }^{49}$ (v.1.18). Data from a single crystal of C102 Fab-RBD complex were indexed and integrated using XIA $2^{50} \mathrm{v} \cdot 0.3 .8$ implementing DIALS ${ }^{51,52}$ v.2.2 and merged using AIMLESS in CCP4 $4^{48}$. For C110 Fab and $\mathrm{C} 121 \mathrm{Fabs}$, structures were determined by molecular replacement in PHASER $^{53}$ v.2.8.2 using the coordinates for B38 (PDB 7BZ5) or an inferred germline form of the HIV-1 NAb IOMA ${ }^{54}$ inferred germline (unpublished), respectively, after removing CDR loops as a search model. For C002 Fab, C102 Fab, C102 Fab-RBD and C135 Fab, structures were determined by molecular replacement in PHASER $^{53}$ using B38 Fab coordinates (PDB 7BZ5) after trimming heavy and light chain variable domains using Sculptor ${ }^{55}$ v.2.0 (and for the C102 Fab-RBD data, also RBD coordinates from PDB code 7BZ5) as search models. Coordinates were refined using Phenix ${ }^{49}$ and cycles of manual building in $\operatorname{Coot}^{56}$ (Supplementary Table 1).

\section{Cryo-EM sample preparation}

Purified Fabs were mixed with the SARS-CoV-2 S 2P trimer $^{35}$ or SARS-CoV-2S 6P trimer $^{36}$ (1.1:1 molar ratio Fab per protomer) to a final Fab-S complex concentration of $2-3 \mathrm{~g} \mathrm{~m}^{-1}$ and incubated on ice for $30 \mathrm{~min}$. Immediately before deposition of $3 \mu \mathrm{l}$ of complex onto a 300 mesh, 1.2/1.3 UltrAuFoil grid (Electron Microscopy Sciences) that had been freshly glow-discharged for $1 \mathrm{~min}$ at $20 \mathrm{~mA}$ using a PELCO easiGLOW (Ted Pella), a $0.5 \%$ (w/v) octyl-maltoside, fluorinated solution (Anatrace) was added to each sample to a final concentration of $0.02 \%$. Samples were vitrified in $100 \%$ liquid ethane using a Mark IV Vitrobot (Thermo Fisher) after blotting at $22^{\circ} \mathrm{C}$ and $100 \%$ humidity for $3 \mathrm{~s}$ with Whatman No. 1 filter paper.

\section{Cryo-EM data collection and processing}

Single-particle cryo-EM data were collected on a Titan Krios transmission electron microscope (Thermo Fisher) operating at $300 \mathrm{kV}$ for all Fab-S complexes except for C144-S, which was collected on a Talos Arctica (Thermo Fisher) operating at $200 \mathrm{kV}$. Movies were collected using SerialEM v.3.7 automated data collection software ${ }^{57}$ with beam-image shift over a 3-by-3 pattern of $1.2 \mu \mathrm{m}$ holes with 1 exposure per hole. Movies were recorded in super-resolution mode on a K3 camera (Gatan) for the C144-S dataset on the Arctica (0.435 $\mathrm{A}$ per pixel) or on a K3 behind BioQuantum energy filter (Gatan) with a $20 \mathrm{eV}$ slit on the Krios ( $0.418 \AA$ per pixel) for all other datasets. Data collections parameters are summarized in Supplementary Table 2. In general, the dat- processing workflow described below was performed for all datasets in cryoSPARC v. $2.15^{58}$.

Cryo-EM movies were patch motion corrected for beam-induced motion including dose weighting within cryoSPARC ${ }^{58}$ after binning super-resolution movies. The non-dose-weighted images were used to estimate CTF parameters using CTFFIND $4^{59}$ v.4.1.14 or with cryoSPARC implementation of the Patch CTF job, and micrographs with power spectra that showed poor CTF fits or signs of crystalline ice were discarded. A subset of images were randomly selected and used for reference-free particle picking using Blob picker in cryoSPARC ${ }^{58}$. Particles were subjected to 2D classification and the best class averages that represented different views were used to generate $3 \mathrm{ab}$ initio models. The particles from the best classes were used in another 2D classification job, and the best set of unique views was used as templates for particle picking on the full set of images. Initial particle stacks were extracted, downsampled twice, and used in heterogeneous refinement against the three ab initio volumes generated with the smaller dataset (ab initio volumes used were interpreted as a Fab-S complex, free Fab or dissociated S protomers, and junk/noise class). Particles assigned to the Fab-S volume were further cleaned via iterative rounds of 2D classification to select class averages that displayed unique views and secondary structural elements. Resulting particle stacks were homogenously refined before being split into nine individual exposure groups based upon collection holes. Per particle CTF and aberration corrections were performed and the resulting particles further 3D refined. Additional processing details are summarized in Supplementary Table 2.

Given the known heterogeneity of spike trimers ${ }^{20,21}$, homogenously refined particles were used for $3 D$ classification in cryoSPARC $^{58}$ (ab initiojob: $k=4$ classes, class similarity $=0.3$ ). This typically resulted in one or two majority Fab-S complexes, with the other minority populated classes representing junk or unbound $S$ trimer. Particles from the good class(es) were further subjected to 3D classification (ab initiojob: $k=4$, class similarity $=0.7$ ) to attempt to separate various Fab-S complex states. If several states were identified (as observed for the C002-S and C121-S complexes), particles were heterogeneously refined, followed by re-extraction without binning $(0.836 \AA$ per pixel) before homogeneous refinement of individual states. For all other datasets, most particles represented one state that was homogenously refined after re-extraction without binning. 
Particle stacks for individual states were non-uniform refined with $C 1$ symmetry and a dynamic mask. To improve resolution at the Fab-RBD interfaces, volumes were segmented in Chimera $^{60}$ and the regions corresponding to the NTD and RBD domains of the S1 subunit and the Fab VH-VL domains were extracted and used to generate a soft mask (5-pixel extension, 10-pixel soft cosine edge). Local refinements with the mask resulted in modest improvements of the Fab-RBD interface, which allowed for fitting and refinement of this region. The particles were then subjected to CTF refinement and aberration correction, followed by a focused, non-uniform refinement with polished particles imposing $C 1$ symmetry (except for the $\mathrm{C} 144-\mathrm{S}$ complex, in which $C 3$ symmetry was used). Final overall resolutions were according to the gold-standard FSC ${ }^{61}$. Details of overall resolution and locally refined resolutions according to the gold-standard $\mathrm{FSC}^{61}$ can be found in Supplementary Table 2 .

\section{Cryo-EM structure modelling and refinement}

Coordinates for initial complexes were generated by docking individual chains from reference structures into cryo-EM density using UCSF Chimera $^{62}$ v.1.13. The following coordinates were used: SARS-CoV-2 S trimers: $\mathrm{PDB}$ codes $6 \mathrm{VXX}, 6 \mathrm{VYB}$ and $6 \mathrm{XKL}$, up RBD conformations: $\mathrm{PDB}$ codes 7BZ5 or 6W41, and unbound C102, C002, C110, C121, C135 Fab structures (this study) (Supplementary Table 1). Initial models were then refined into cryo-EM maps using one round of rigid body refinement followed by real space refinement. Sequence-updated models were built manually in $\operatorname{Coot}^{56}$ v.0.8.9 and then refined using iterative rounds of refinement in $\operatorname{Coot}^{56}$ and Phenix ${ }^{49}$. Glycans were modelled at potential $N$-linked glycosylation sites in $\operatorname{Coot}^{56}$ using 'blurred' maps processed with a variety of B-factors ${ }^{63}$. Validation of model coordinates was performed using MolProbity ${ }^{64}$ (Supplementary Table 2).

\section{Structural analyses}

CDR lengths were calculated based on IMGT definitions ${ }^{32}$. Structure figures were made with PyMOL (v.2.2 Schrodinger, LLC) or UCSF ChimeraX ${ }^{60}$ v.1.0. Local resolution maps were calculated using cryOSPARC v.2.15 ${ }^{58}$. Buried surface areas were calculated using PDBePISA v.1.48 ${ }^{65}$ and a $1.4 \AA$ probe. Potential hydrogen bonds were assigned as interactions that were less than $4.0 \AA$ and with an A-D-H angle above $90^{\circ}$. Potential van der Waals interactions between atoms were assigned as interactions that were less than $4.0 \AA$. Hydrogen bond and van der Waals interaction assignments are tentative due to resolution limitations. r.m.s.d. calculations following pairwise $\mathrm{C} \alpha$ alignments were done in PyMOL without rejecting outliers. Criteria for epitope assignments are described in figure legends.

To evaluate whether intra-spike crosslinking by an IgG binding to a single spike trimer was possible (Extended Data Table1), we first measured the $\mathrm{C} \alpha$ distance between a pair of residues near the $C$ termini of adjacent Fab $\mathrm{CH} 1$ domains (residue 222 of the heavy chain on each Fab) (Extended Data Fig. 5h). We compared this distance to the analogous distances in crystal structures of intact IgGs ( $42 \AA$ A PDB code $1 \mathrm{HZH} ; 48 \AA$, PDB code IIGY; $52 \AA$, PDB code 1IGT). To account for potential influences of crystal packing in these measurements, as well as flexibility in the $\mathrm{VH}-\mathrm{VL} / \mathrm{CH} 1-\mathrm{CL}$ elbow bend angle and uncertainties in $\mathrm{CH} 1-\mathrm{CL}$ domain placement in $\mathrm{Fab}-\mathrm{S}$ cryo-EM structures, we set a cut-off of $\leq 65 \AA$ for this measured distance as possibly allowing for a single IgG to include both Fabs. Entries in the 'potential IgG intra-spike binding' column in Extended Data Table 1 are marked 'no' if all of the adjacent Fabs in cryo-EM classes of that structure are separated by more than $65 \AA$ for this measured distance. Entries in the 'potential IgG intra-spike binding' column in Extended Data Table 1 are marked as 'yes' if at least one pair of the adjacent Fabs in cryo-EM classes of that structure are separated by $\leq 65 \AA$ for this measured distance.

\section{SPR binding experiments}

SPR experiments were performed using a Biacore T200 instrument (GE Healthcare). IgGs were immobilized on a CM5 chip by primary amine chemistry (Biacore manual) to a final response level of approximately 3,000 resonance units. Concentration series of the original SARS-CoV-2 RBD and RBD mutants (six fourfold dilutions starting from a top concentration of $1,000 \mathrm{nM}$ ) were injected at a flow rate of $30 \mu \mathrm{lmin}$ over immobilized IgGs for a contact time of $60 \mathrm{~s}$, followed by an injection of 0.01 M HEPES, pH 7.4, 0.15 M NaCl, 3 mMEDTA, $0.005 \%$ (v/v) surfactant $\mathrm{P} 20$ buffer for a dissociation time of $300 \mathrm{~s}$. Binding reactions were allowed to reach equilibrium, and $K_{\mathrm{d}}$ values were calculated from the ratio of association and dissociation rates $\left(K_{\mathrm{d}}=k_{\mathrm{d}} / k_{\mathrm{a}}\right)$ derived from a 1:1 binding model (C002, C102, C105, C110 and C119 (except for C119 mutant $\mathrm{E} 484 \mathrm{~K})$ ), C121, C135 and C144), or from a two-state binding model $\left(K_{\mathrm{d}}=k_{\mathrm{d}} 1 / k_{\mathrm{a}} 1 \times k_{\mathrm{d}} 2 /\left[k_{\mathrm{d}} 2+k_{\mathrm{a}} 2\right]\right)(\mathrm{C} 104$ and C119 mutant E484K). Kinetic constants were calculated using Biacore T200 Evaluation Software v.3.2 using a global fit to all curves in each dataset. Flow cells were regenerated with $10 \mathrm{mM}$ glycine, $\mathrm{pH} 2.0$, at a flow rate of $90 \mu \mathrm{min}^{-1}$.

\section{Polyreactivity assays}

IgGs were evaluated for off-target interactions by measuring binding to baculovirus extracts containing non-specific proteins and lipids as described $^{59}$. The assays were automated on a Tecan Evo2 liquid handling robot fitted with a Tecan Infinite M1000 plate reader capable of reading luminescence. Maxisorb 384-well plates (Nunc) were adsorbed overnight with a $1 \%$ preparation of recombinant baculovirus particles generated in Sf9 insect cells ${ }^{66}$. The adsorbed plate was blocked with $0.5 \%$ bovine serum albumin (BSA) in PBS, then incubated with $20 \mu \mathrm{l}$ of a $1.0 \mu \mathrm{g} \mathrm{ml}^{-1}$ solution of IgG in PBS for $3 \mathrm{~h}$. Polyreactivity was quantified by detecting bound IgG using an HRP-conjugated anti-human IgG secondary antibody (SouthernBiotech) at a 1:5,000 dilution and SuperSignal ELISA Femto Maxiumum Sensitivity Substrate (Thermo Scientific). RLUs were measured at $475 \mathrm{~nm}$ in the integrated plate reader. Engineered human anti-HIV-1 IgGs previously demonstrated to exhibit high levels of polyreactivity (NIH45-46(G54W) and 45-46m2 $)^{60,61}$ were used as positive controls. NIH45-46, which exhibited intermediate polyreactivity $^{62}$, was also evaluated for comparisons. Negative control IgGs with low polyreactivity included the human HIV-1 antibodies $\mathrm{N}^{63}$ and $3 \mathrm{BNC} 117^{62}$ and BSA. RLU values were plotted in GraphPad Prism v8.4.3 and presented as the mean and standard deviation of triplicate measurements ( $n=3$ biological replicates) with results for individual experiments shown as circles in Extended Data Fig. 1i.

\section{Reporting summary}

Further information on research design is available in the Nature Research Reporting Summary linked to this paper.

\section{Data availability}

The atomic models generated from X-ray crystallographic studies of the C102-RBD complex, C102 Fab, C002 Fab, C110 Fab, C121 Fab and C135 Fab have been deposited at the Protein Data Bank (PDB) under accession codes 7K8M, 7K8N, 7K8O, 7K8P, 7K8Q and 7K8R, respectively. The atomic models and cryo-EM maps generated from cryo-EM studies of the C002-S 2P (state 1), C002-S 2P (state 2), C104-S 2P, C110-S 2P, C119-S 2P, C121-S 2P (state 1), C121-S 2P (state 2), C135-S 2P and $\mathrm{C} 144-\mathrm{S} 6 \mathrm{P}$ complexes have been deposited at the PDB and the Electron Microscopy Data Bank (EMDB) under the following accession codes: $\mathrm{PDB} 7 \mathrm{~K} 8 \mathrm{~S}, 7 \mathrm{~K} 8 \mathrm{~T}, 7 \mathrm{~K} 8 \mathrm{U}, 7 \mathrm{~K} 8 \mathrm{~V}, 7 \mathrm{~K} 8 \mathrm{~W}, 7 \mathrm{~K} 8 \mathrm{X}, 7 \mathrm{~K} 8 \mathrm{Y}, 7 \mathrm{~K} 8 \mathrm{Z}$ and 7K90; EMD EMD-22729, EMD-22730, EMD-22731, EMD-22372, EMD-22733, EMD-22734, EMD-22735, EMD-22736 and EMD-22737.

\footnotetext{
45. Scharf, L. et al. Broadly neutralizing antibody 8ANC195 recognizes closed and open states of HIV-1 Env. Cell 162, 1379-1390 (2015).

46. Schoofs, T. et al. Broad and potent neutralizing antibodies recognize the silent face of the HIV envelope. Immunity 50, 1513-1529 (2019).

47. Kabsch, W. XDS. Acta Crystallogr. D 66, 125-132 (2010).

48. Winn, M. D. et al. Overview of the CCP4 suite and current developments. Acta Crystallogr. D 67, 235-242 (2011).
} 


\section{Article}

49. Adams, P. D. et al. PHENIX: a comprehensive Python-based system for macromolecular structure solution. Acta Crystallogr. D 66, 213-221 (2010).

50. Winter, G. xia2: an expert system for macromolecular crystallography data reduction. J. Appl. Crystallogr. 43, 186-190 (2010).

51. Beilsten-Edmands, J. et al. Scaling diffraction data in the DIALS software package: algorithms and new approaches for multi-crystal scaling. Acta Crystallogr. D. 76, 385-399 (2020).

52. Winter, G. et al. DIALS: implementation and evaluation of a new integration package. Acta Crystallogr. D 74, 85-97 (2018)

53. McCoy, A. J. et al. Phaser crystallographic software. J. Appl. Crystallogr. 40, 658-674 (2007).

54. Gristick, H. B. et al. Natively glycosylated HIV-1 Env structure reveals new mode for antibody recognition of the CD4-binding site. Nat. Struct. Mol. Biol. 23, 906-915 (2016).

55. Bunkóczi, G. \& Read, R. J. Improvement of molecular-replacement models with Sculptor. Acta Crystallogr. D 67, 303-312 (2011)

56. Emsley, P., Lohkamp, B., Scott, W. G. \& Cowtan, K. Features and development of Coot. Acta Crystallogr. D 66, 486-501 (2010).

57. Mastronarde, D. N. Automated electron microscope tomography using robust prediction of specimen movements. J. Struct. Biol. 152, 36-51 (2005).

58. Punjani, A., Rubinstein, J. L., Fleet, D. J. \& Brubaker, M. A. cryoSPARC: algorithms for rapid unsupervised cryo-EM structure determination. Nat. Methods 14, 290-296 (2017).

59. Rohou, A. \& Grigorieff, N. CTFFIND4: fast and accurate defocus estimation from electron micrographs. J. Struct. Biol. 192, 216-221 (2015).

60. Goddard, T. D. et al. UCSF ChimeraX: meeting modern challenges in visualization and analysis. Protein Sci. 27, 14-25 (2018).

61. Bell, J. M., Chen, M., Baldwin, P. R. \& Ludtke, S. J. High resolution single particle refinement in EMAN2.1. Methods 100, 25-34 (2016).

62. Goddard, T. D., Huang, C. C. \& Ferrin, T. E. Visualizing density maps with UCSF Chimera. J. Struct. Biol. 157, 281-287 (2007).

63. Terwilliger, T. C., Adams, P. D., Afonine, P. V. \& Sobolev, O. V. A fully automatic method yielding initial models from high-resolution cryo-electron microscopy maps. Nat. Methods 15, 905-908 (2018).

64. Chen, V. B. et al. MolProbity: all-atom structure validation for macromolecular crystallography. Acta Crystallogr. D 66, 12-21 (2010).

65. Krissinel, E. \& Henrick, K. Inference of macromolecular assemblies from crystalline state. J. Mol. Biol. 372, 774-797 (2007)

66. Davis, M. I., Bennett, M. J., Thomas, L. M. \& Bjorkman, P. J. Crystal structure of prostate-specific membrane antigen, a tumor marker and peptidase. Proc. Natl Acad. Sci. USA 102, 5981-5986 (2005).

67. Liu, H. et al. Cross-neutralization of a SARS-CoV-2 antibody to a functionally conserved site is mediated by avidity. Preprint at https://doi.org/10.1101/2020.08.02.233536 (2020).

68. Zhou, D. et al. Structural basis for the neutralization of SARS-CoV-2 by an antibody from a convalescent patient. Nat Struct. Mol. Biol. 27, 950-958 (2020).

69. Piccoli, L. et al. Mapping neutralizing and immunodominant sites on the SARS-CoV-2 spike receptor-binding domain by structure-guided high-resolution serology. Cell $\mathbf{1 8 3}$ 1024-1042 (2020)
70. Dunbar, J. et al. SAbDab: the structural antibody database. Nucleic Acids Res. 42, D1140-D1146 (2014).

Acknowledgements We thank J. Vielmetter, P. Hoffman, and the Protein Expression Center in the Beckman Institute at Caltech for expression assistance, J. Vielmetter and J. Keeffe for setting up automated polyreactivity assays, J. Keeffe for construct design, and N. Koranda for help with cloning and protein purification. Electron microscopy was performed in the Caltech Beckman Institute Resource Center for Transmission Electron Microscopy with assistance from S. Chen. We thank the Gordon and Betty Moore and Beckman Foundations for gifts to Caltech to support the Molecular Observatory (J. Kaiser, director), and S. Russi, A. Cohen and C. Smith and the beamline staff at SSRL for data collection assistance. Use of the Stanford Synchrotron Radiation Lightsource, SLAC National Accelerator Laboratory, is supported by the US Department of Energy, Office of Science, Office of Basic Energy Sciences under contract no. DE-AC02-c76SF00515. The SSRL Structural Molecular Biology Program is supported by the DOE Office of Biological and Environmental Research, and by the National Institutes of Health, National Institute of General Medical Sciences (P41GM103393). The contents of this publication are solely the responsibility of the authors and do not necessarily represent the official views of NIGMS or NIH. This work was supported by NIH grant P01-Al138938-S1 (P.J.B. and M.C.N.), the Caltech Merkin Institute for Translational Research (P.J.B.), NIH grant P50 8 P50 Al150464-13 (P.J.B.), and a George Mason University Fast Grant (P.J.B.). C.O.B was supported by the Hanna Gray Fellowship Program from the Howard Hughes Medical Institute and the Postdoctoral Enrichment Program from the Burroughs Wellcome Fund. M.C.N. is a Howard Hughes Medical Institute Investigator.

Author contributions C.O.B., M.C.N., A.P.W. and P.J.B. conceived the study and analysed data D.F.R. and M.C.N. provided monoclonal antibody sequences and plasmids derived from COVID-19-convalescent donors. C.O.B. and K.H.T. performed protein purifications and C.O.B. assembled complexes for cryo-EM and X-ray crystallography studies. C.O.B. performed cryo-EM and interpreted structures with assistance from M.E.A., K.A.D., S.R.E., A.G.M. and N.G.S. C.A.J. and C.O.B. performed and analysed crystallographic structures, with refinement assistance from M.E.A. and K.M.D. Y.E.L. performed polyreactivity assays. H.B.G. performed and analysed SPR experiments. A.P.W. analysed antibody sequences. C.O.B., M.C.N., A.P.W. and P.J.B. wrote the paper with contributions from other authors.

Competing interests The Rockefeller University has filed a provisional patent application in connection with this work on which D.F.R. and M.C.N. are inventors (US 63/021,387).

\section{Additional information}

Supplementary information is available for this paper at https://doi.org/10.1038/s41586-0202852-1.

Correspondence and requests for materials should be addressed to P.J.B.

Peer review information Nature thanks the anonymous reviewer(s) for their contribution to the peer review of this work.

Reprints and permissions information is available at http://www.nature.com/reprints 


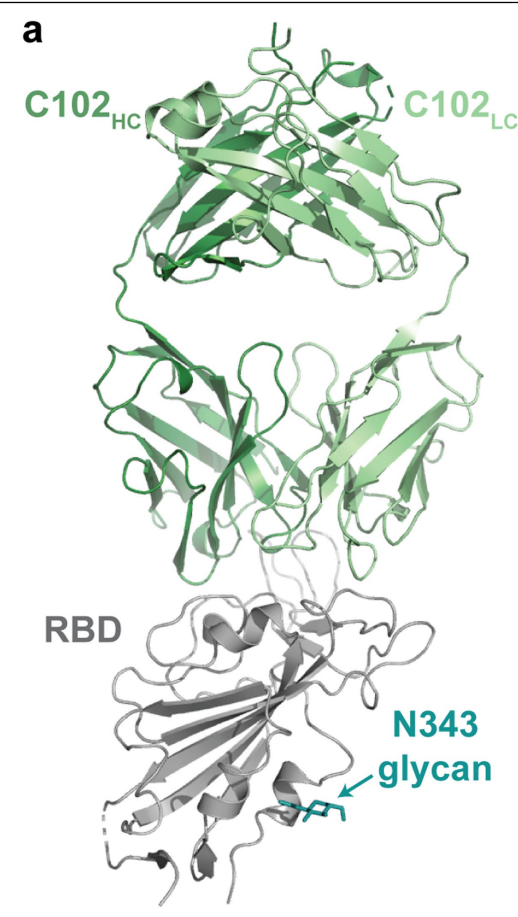

f

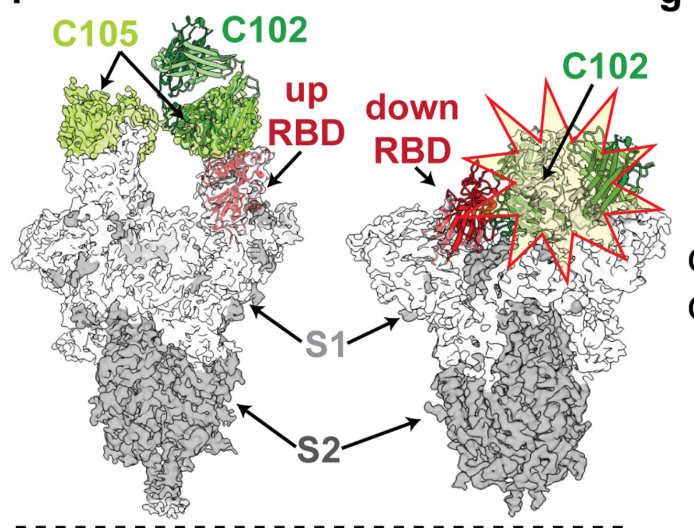

Viral Membrane

h C144 $_{\text {COVA2-39 }}$ COVA2-39 ${ }_{\mathrm{HC}}$ i

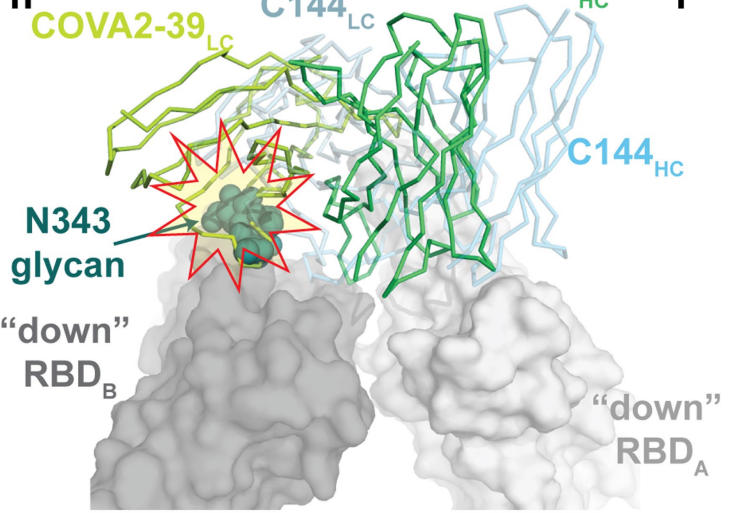

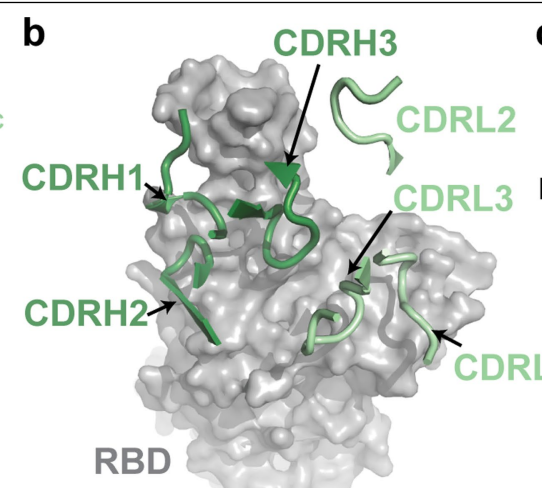

d

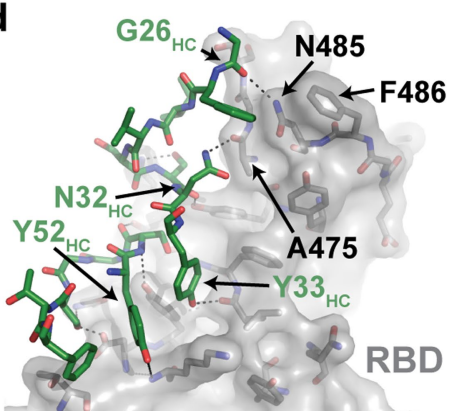

g
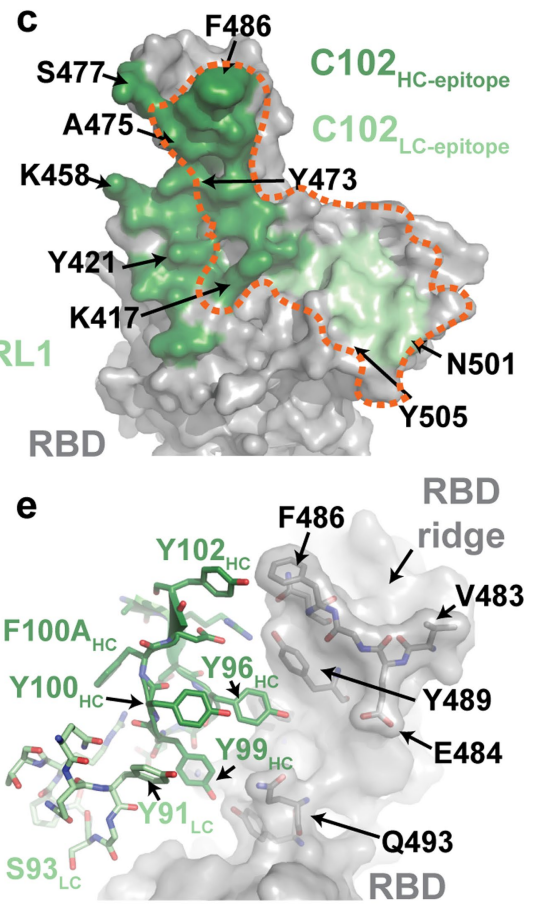

Antibody

CDRH3 Sequence

C144 AREGEVEGYNDFWSGYSRDRYYFDY

C102 ARDYGD-------------Y-YFDY

C105 ARGEGWE------------L-PYDY

COVA2-04 ARDLERA-----------G-GMDV

COVA2-39 ARAHVDTAM--------VESGAFDI

B38 ARE-------------AYGMDV

CB6 ARVLPMY-----------GDYLDY

CC12.1 ARDLD-V-----------Y-GLDV

CC12.3 ARDFG-D-----------F-YFDY

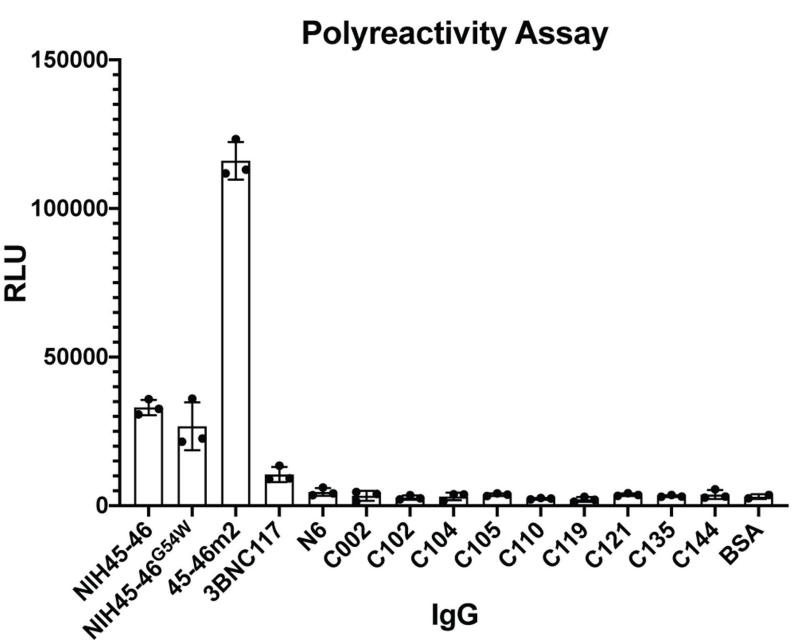

Extended Data Fig. 1 |See next page for caption. 


\section{Article}

Extended Data Fig. 1 |X-ray structure and epitope mapping of VH3-53 NAb C102. a, X-ray structure of the $\mathrm{C} 102 \mathrm{Fab}-\mathrm{RBD}_{331-518}$ complex. b, C102 CDR loops mapped on the RBD surface.c, Surface representation of C102 epitope coloured by $\mathrm{C} 102$ heavy chain (dark green) and light chain (light green) interactions. d, e, CDRH1, CDRH2 (d) and CDRH3 (e) interactions with RBD residues. Potential hydrogen-bond contacts are illustrated as dashed lines. f, Left, overlay of C102-RBD crystal structure (cartoon) with C105-S trimer cryoEM density (PDB 6XCM, EMD-22127) illustrating conserved binding to RBD epitope in an up conformation. Right, the $\mathrm{C} 102$ epitope is sterically occluded when aligned to a down RBD conformation (red and yellow star). SARS-CoV-2S domains are dark grey (S2 domain) and light grey (S1 domain); the C105 Fab is yellow-green.g, Alignment of selected CDRH3 sequences for $\mathrm{VH3}$-53- or VH3-66-encoded SARS-CoV-2 neutralizing antibodies (IMGT definition ${ }^{32}$ ). h, Overlay of NAb COVA2-39 $\mathrm{Fab}^{38}$ (lime green and lemon, from COVA2-39-RBD structure, PDB 7JMP) and C144 Fab (blue, from C144-S structure) aligned on a $\mathrm{RBD}_{\mathrm{A}}$ of $\mathrm{C} 144$ epitope. COVA2-39 adopts a distinct conformation relative to the C102-like VH3-53-encoded short CDRH3 NAb class and to $\mathrm{C} 144$, recognizing its RBD epitope only in an up RBD conformations owing to steric clashes (red and yellow star) with the N343-associated glycan on the adjacent RBD. i, Polyreactivity assay. IgGs were evaluated for binding to baculovirus extracts to assess nonspecific binding. Polyreactive positive control IgGs were NIH45-46, 45-46 ${ }^{\mathrm{G} 54 \mathrm{~W}}$ and $45-46 \mathrm{~m} 2$. Negative controls were bovine serum albumin (BSA) and IgGs N6 and 3BNC117. Relative light unit (RLU) values are presented as the mean and s.d. of triplicate measurements ( $n=3$ biological replicates) with results for individual experiments shown as circles. 

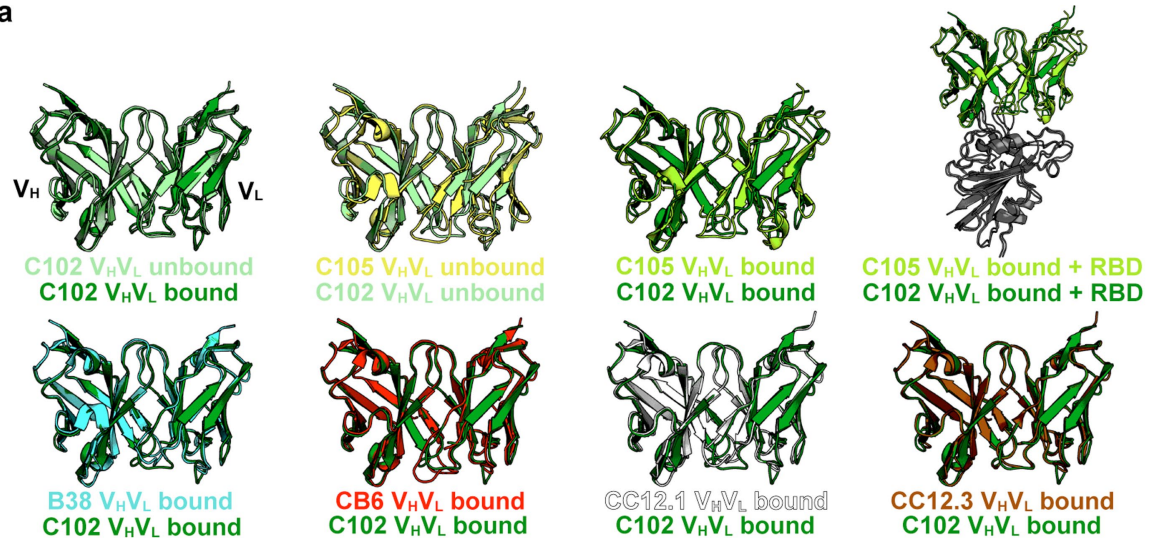

\begin{tabular}{|cccccc|}
\hline \multicolumn{6}{|c|}{ Alignments of $\mathbf{V}_{\mathbf{H}} \mathbf{V}_{\mathbf{L}}$ Domains of $\mathbf{V H 3}$-53/short CDRH3 NAbs } \\
\hline Structure 1 & Structure 2 & \# Ca atoms & RMSD $(\mathbf{A})$ PDB Structure 1 & PDB Structure 2 \\
C102 unbound & C102 bound & 225 & 0.9 & This study & This study \\
C105 unbound & C102 unbound & 220 & 2.0 & 6 XCA & This study \\
C105 bound & C102 bound & 220 & 3.0 & 6 XCM & This study \\
C105 bound + RBD & C102 bound + RBD & 403 & 2.4 & 6 XCM & This study \\
B38 bound & C102 bound & 222 & 1.1 & $7 B Z 5$ & This study \\
CB6 bound & C102 bound & 223 & 1.2 & 7 C01 & This study \\
CC12.1 bound & C102 bound & 224 & 1.1 & 6 XC2 & This study \\
CC12.3 bound & C102 bound & 224 & 0.6 & 6 XC4 & This study \\
\hline
\end{tabular}

b

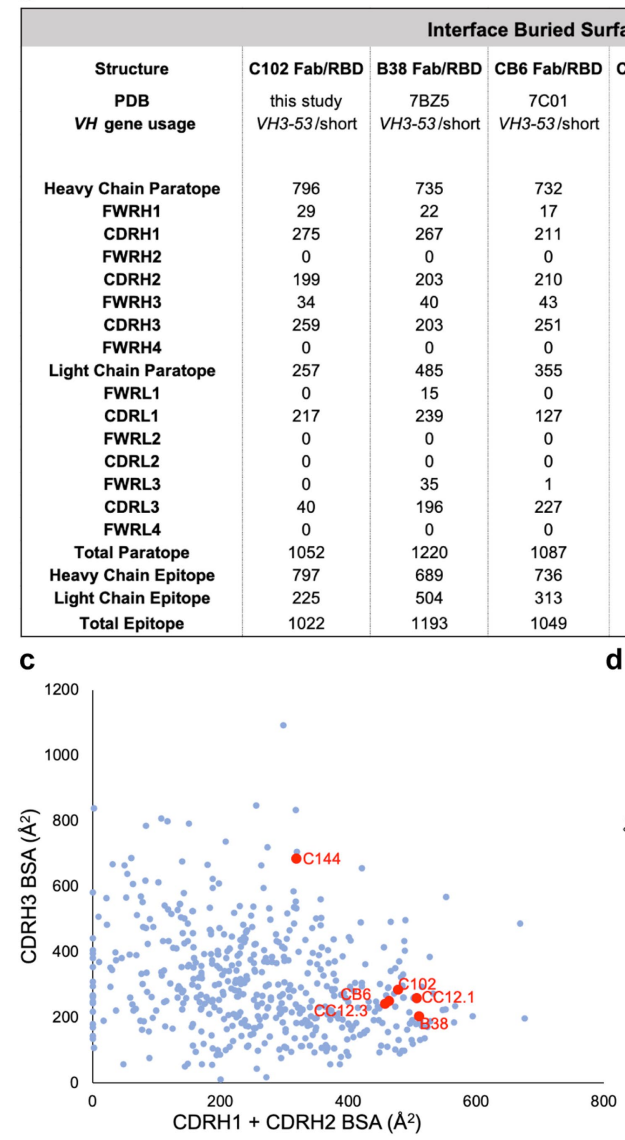

Extended Data Fig. 2 | Overview of $\mathrm{VH3}-53$ / VH3-66-encoded human NAb structures. a, Superimposition of $\mathrm{VH}$ and VL domains of $\mathrm{C} 102$ with other VH3-53- or VH3-66-encoded NAbs (top) and root mean square deviation (r.m.s.d.) calculations (bottom). b, Buried surface area comparisons for the indicated Fab-RBD structures. Buried surface areas were calculated using PDBePISA $^{65}$ and a 1.4 A probe. $\mathbf{c}, \mathbf{d}$, Heavy-chain buried surface areas of anti-SARS-CoV-2 antibodies plotted as CDRH1 (IMGT residues 27-38) plus CDRH2 (residues 56-67*) versus CDRH3 (residues 105-117) (c), and V gene

\begin{tabular}{|c|c|c|c|}
\hline \multicolumn{4}{|l|}{ urface Area $\left(\mathrm{A}^{2}\right)$} \\
\hline \multirow{3}{*}{$\begin{array}{c}\text { CC12.1 Fab/RBD } \\
6 \times C 2 \\
V H 3-53 / \text { short }\end{array}$} & \multirow{3}{*}{$\begin{array}{c}\text { CC12.3 Fab/RBD } \\
6 \text { XC4 } \\
\text { VH3-53/short }\end{array}$} & \multirow{2}{*}{\multicolumn{2}{|c|}{$\begin{array}{c}\text { C144 Fab/RBD } \\
\text { this study } \\
V H 3-53 / \text { long }\end{array}$}} \\
\hline & & & \\
\hline & & RBD A & RBD B \\
\hline 786 & 721 & 707 & 367 \\
\hline 23 & 25 & . & 0 \\
\hline 223 & 217 & 161 & 0 \\
\hline & & & \\
\hline 215 & 204 & 127 & 0 \\
\hline 82 & 42 & 95 & \\
\hline 243 & 233 & 318 & 367 \\
\hline 0 & 0 & 0 & 0 \\
\hline 559 & 164 & 87 & 20 \\
\hline 18 & 1 & 0 & 0 \\
\hline 262 & 111 & 51 & 0 \\
\hline 0 & 0 & 0 & 0 \\
\hline 0 & 0 & 0 & 0 \\
\hline & 0 & 0 & 20 \\
\hline 231 & 52 & 36 & 0 \\
\hline & 0 & 0 & 0 \\
\hline 1345 & 885 & 794 & 387 \\
\hline 763 & 677 & 722 & 330 \\
\hline 574 & 186 & 100 & 22 \\
\hline 1337 & 863 & 822 & 351 \\
\hline
\end{tabular}

d

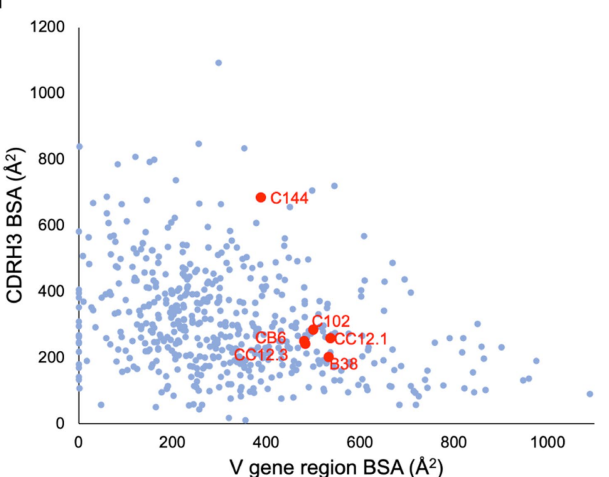

segment region (residues 1-105) versus CDRH3 (residues 106*-117) (d, asterisk indicates differences from IMGT definition). Blue data points represent 501 human antibodies complexed with protein antigens. IMGT-numbered structures (resolutions of $3.5 \AA$ or better) were downloaded from the Structural Antibody Database (SAbDab) ${ }^{70}$ and buried surface area were calculated using PISA $^{65}$. Buried surface areas from antibody structures with identical or near-identical heavy chain sequences were averaged to give a single point on the graph. 


\section{Article}

a

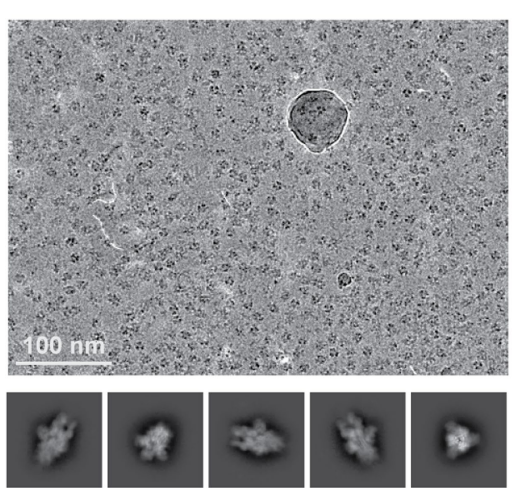

d
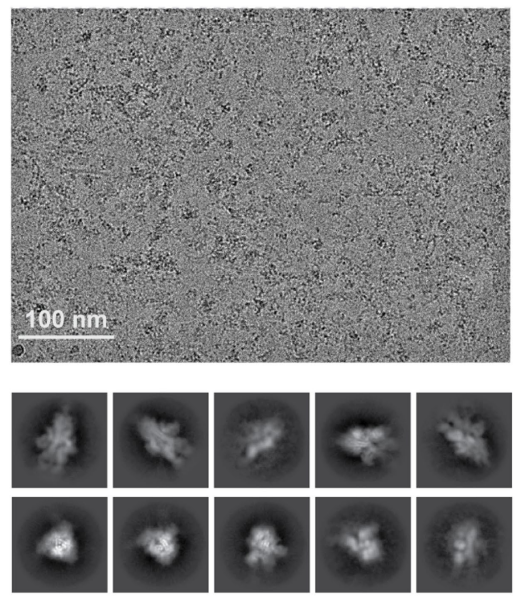

g

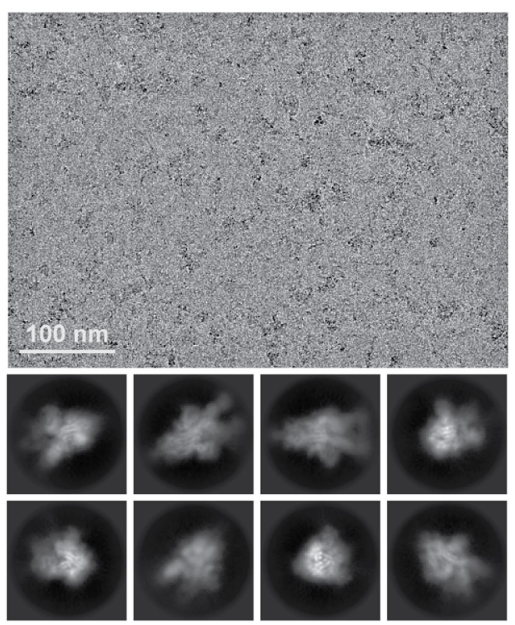

b

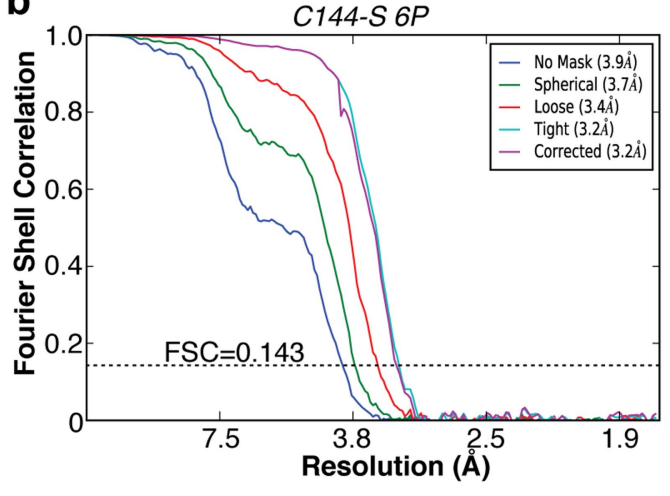

e

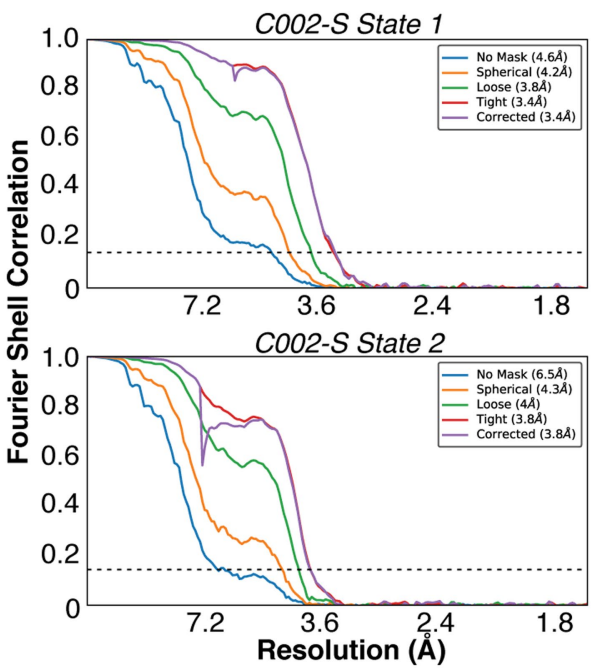

h

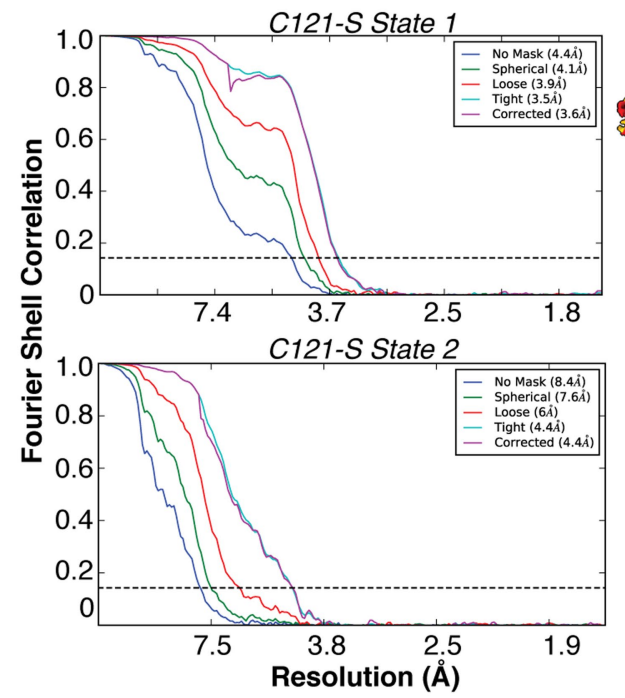

C

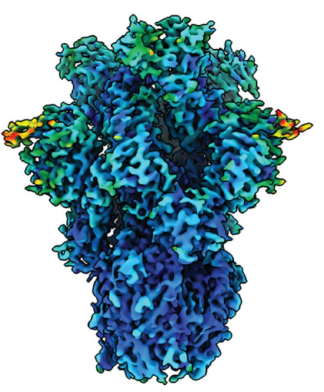

Local Resolution (A)

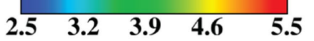

f
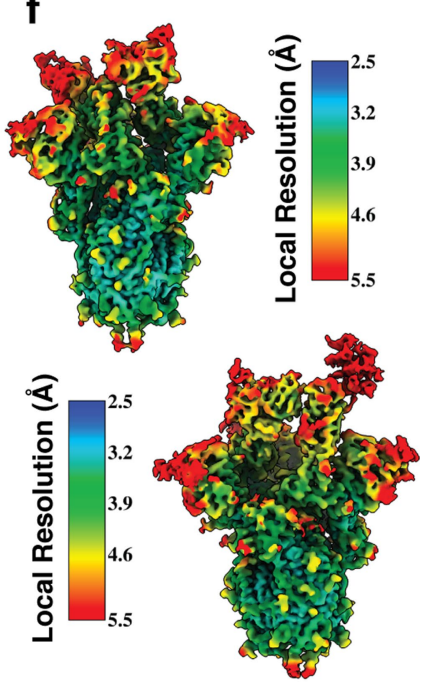

i
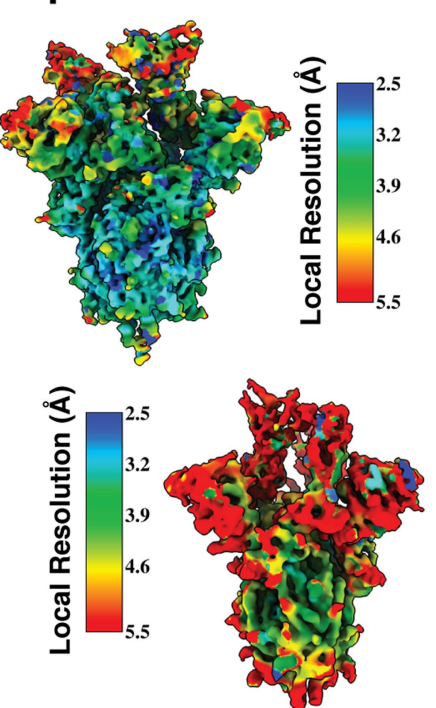

Extended Data Fig. 3 | Cryo-EM data processing and validation for C144-S, C002-S and C121-S complexes. a-i, Representative micrograph selected from total dataset (Supplementary Table 2), 2D class averages, gold-standard Fourier shell correlation (FSC) plots, and local resolution estimations for C144-S 6P (a-c), C002-S 2P (d-f) and C121-S 2P (g-i). Scale bars, $100 \mathrm{~nm}$. For the C002-S dataset, two classes were resolved: state 1, C002 Fabs bound to three down RBDs, and state 2, C002 Fabs bound to two down and one up RBD. For the C121-S2P dataset, two classes were resolved: state 1, C121 Fabs bound to two down and one up RBD and state 2, C121 Fabs bound to one down, two up RBDs. 


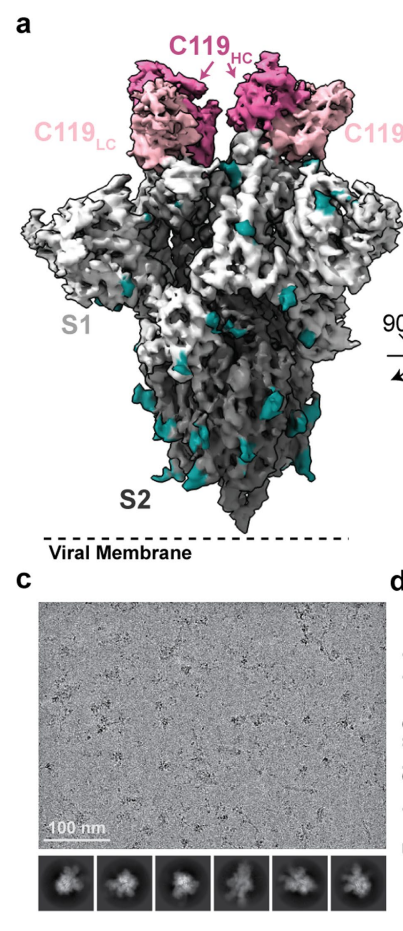

$\mathbf{f}$

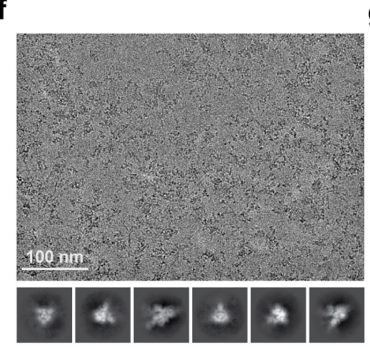

g

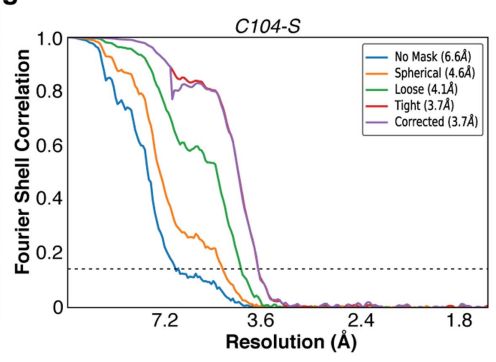

j

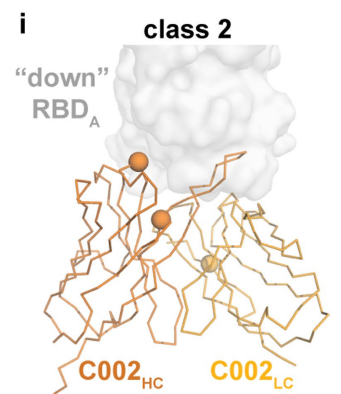

I
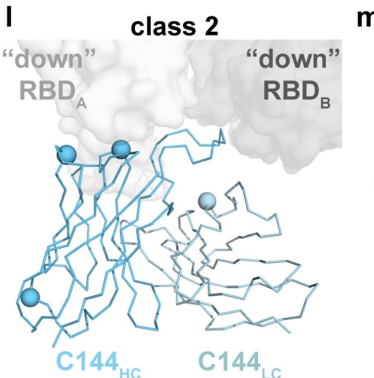
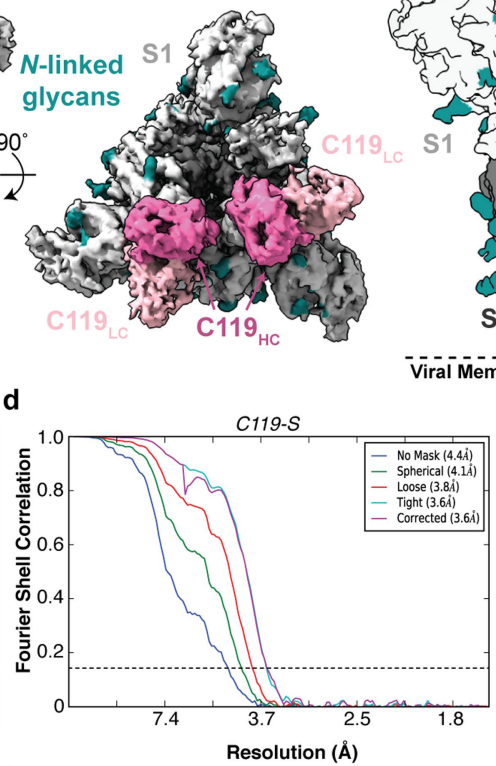

h $\mathbf{k}$

n

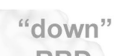

$\mathrm{RBD}_{\mathrm{A}}$

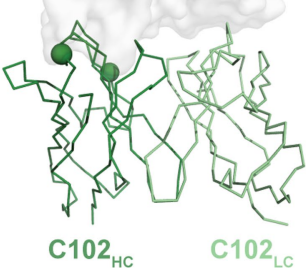

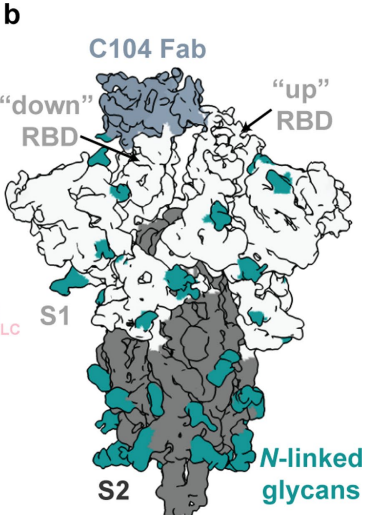

(a)

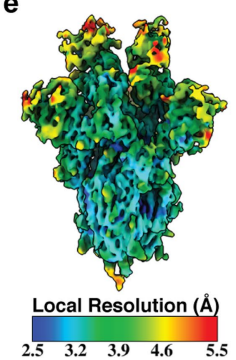

h

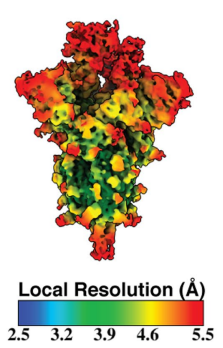

class 2

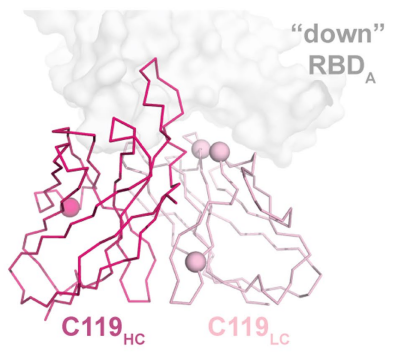

class 3

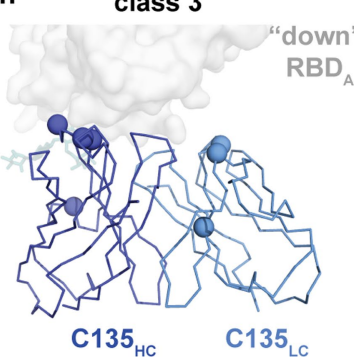

Extended Data Fig. 4 | Cryo-EM processing, validation and reconstruction for C119-S and C104-S complexes. a, 3.6 ^̊ cryo-EM reconstruction for a C119-S trimer complex.b, $3.7 \AA$ cryo-EM reconstruction for a C104-S trimer complex. Representative micrograph selected from the total dataset (Supplementary Table 2), 2D class averages, gold-standard FSC plot, and local resolution estimation for C119-S2P (c-e) and C104-S (f-h). Scale bars, $100 \mathrm{~nm}$.
Both complexes revealed binding of Fabs to both down and up RBD conformations. $\mathbf{i}-\mathbf{n}$, Somatic hypermutations in heavy- and light-chain V gene segments for C002 (i), C121 (j), C119 (k), C144 (1), C102 (m) and C135 (n) are shown as spheres on the antibody $V_{H}$ and VL domains (ribbon representations). The primary RBD epitope is shown as a light grey surface; secondary RBD epitope for C144 is in dark grey. 


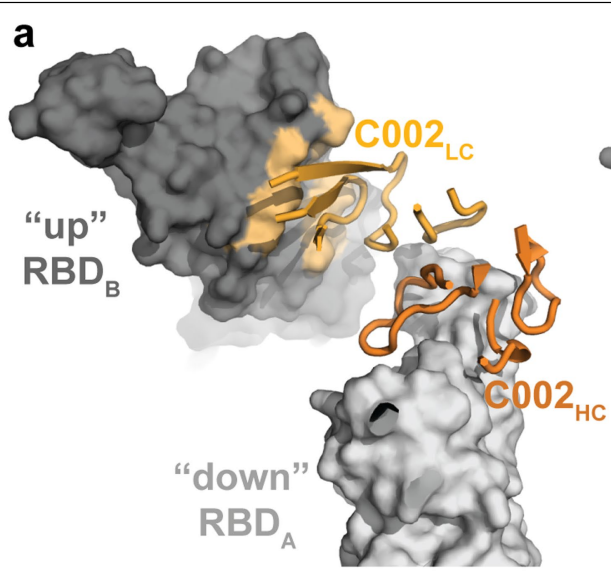

d
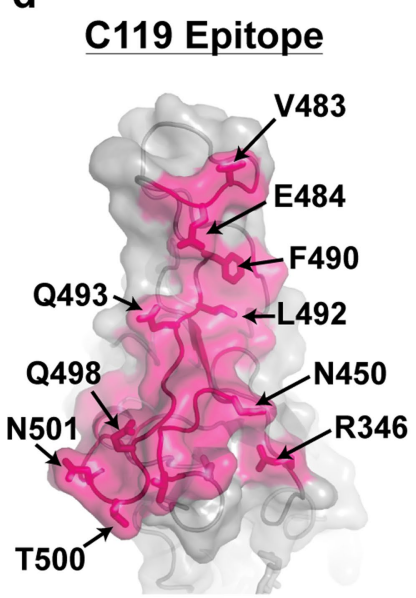

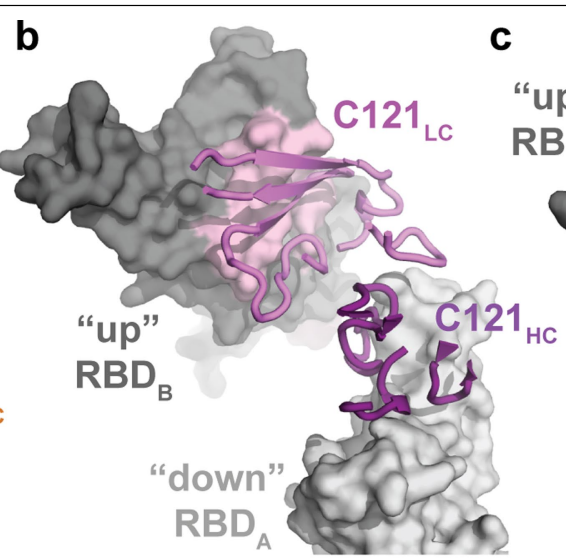

f

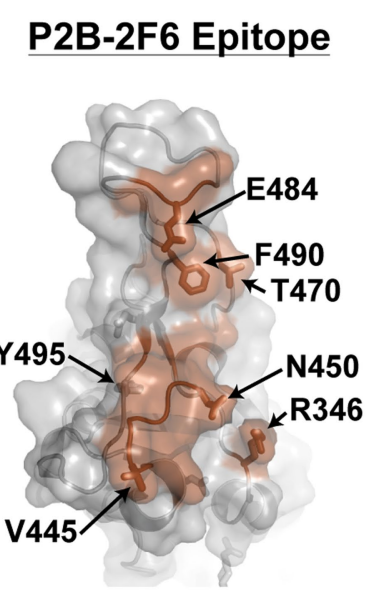

C

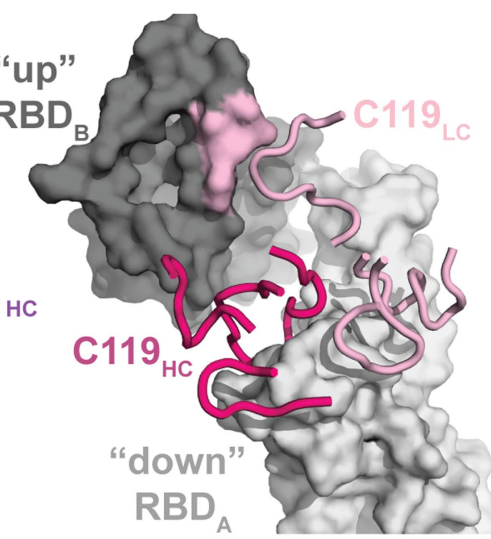

g

h
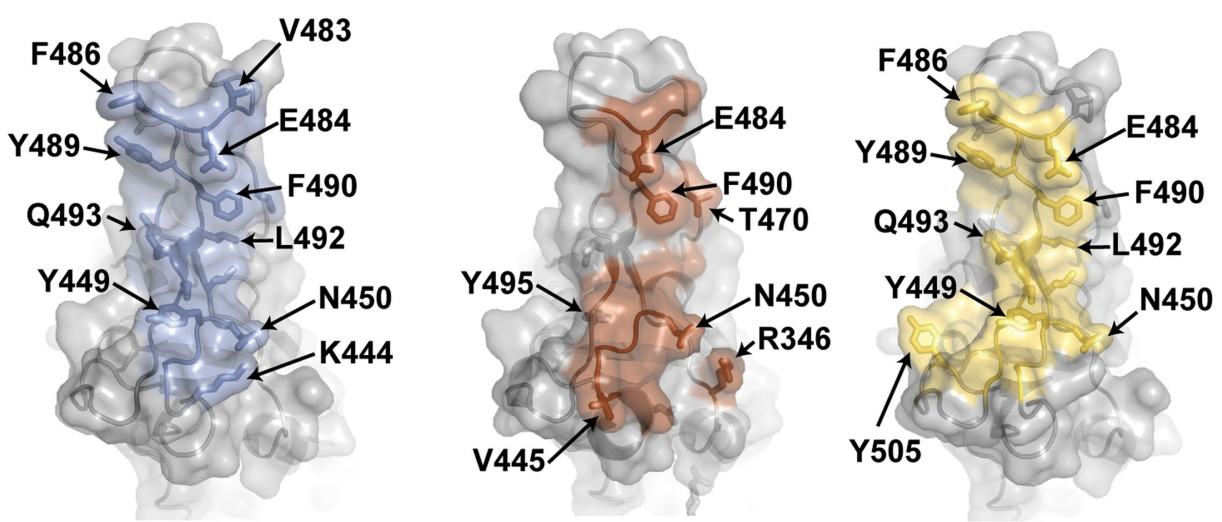

C121-S complex

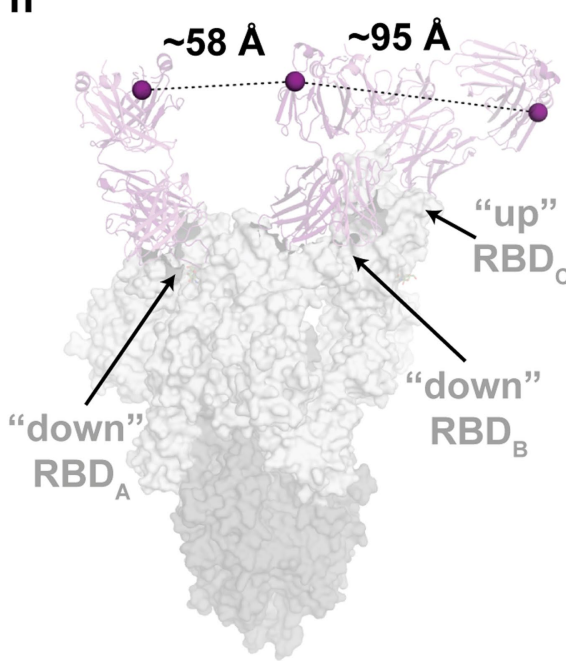

Extended Data Fig. 5 | Primary and secondary epitopes of class 2 human NAbs. a-c, Primary epitopes for $\mathrm{C002}$ (a), $\mathrm{C} 121$ (b), and $\mathrm{C119}$ (c) on down RBD. A secondary epitope is observed if a Fab is bound to an adjacent up RBD for these NAbs. Antibody paratopes are represented as cartoons. A similar interaction in the C104-S structure is not shown owing to low local resolution on the up RBD. d-g, Primary epitopes for $\mathrm{C119}$ (d), C104 (e), P2B-2F6 (f; PDB 7BWJ), and BD23 (g; PDB 7BYR). The existence of secondary epitopes for
P2B-2F6 and BD23 cannot be determined because the P2B-2F6 epitope was determined from a crystal structure with an $\mathrm{RBD}^{27}$, and the BD23-S cryo-EM structure showed only one bound $\mathrm{Fab}^{13} \cdot \mathbf{h}$, Measurement of $\mathrm{C} \alpha$ distance between the $\mathrm{C}$ termini of adjacent $\mathrm{C} 121 \mathrm{CH} 1$ domains (residue 222 of the heavy chain on each Fab). Measurements of this type were used to evaluate whether intra-spike crosslinking by an IgG binding to a single spike trimer was possible for human NAbs in Extended Data Table 1. 


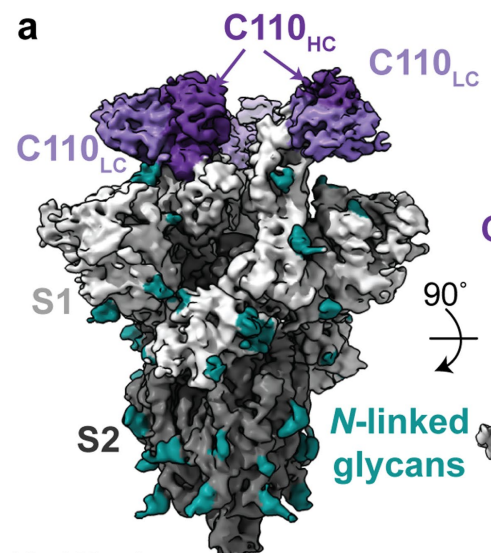

Viral Membrane

C

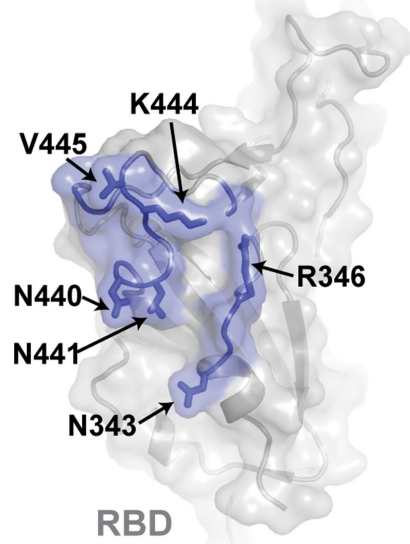

g
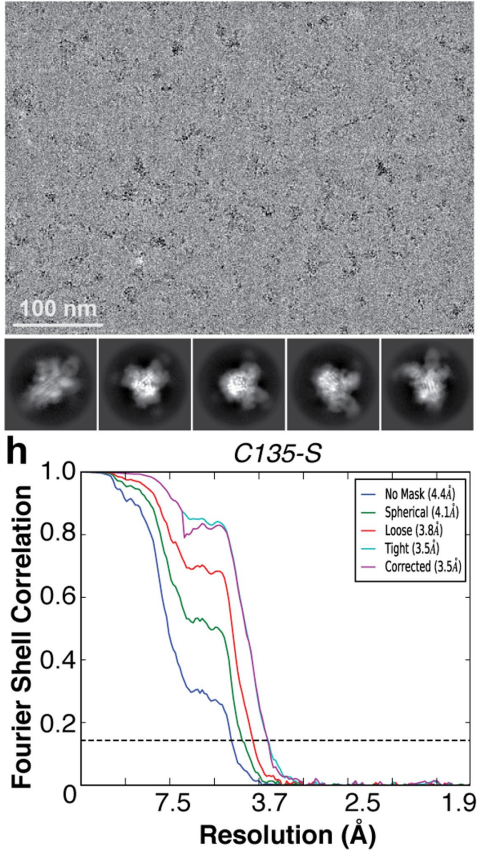

Extended Data Fig. 6 | Cryo-EM structure of C110-S complex and epitope mapping. a, 3.8 Å cryo-EM reconstruction of the C110-S trimer complex.

b, Composite model of C110-RBD (purple and grey, respectively) overlaid with the SARS-CoV-2 NAb REGN-10987 (yellow, PDB 6XDG) and soluble ACE2 (green, PDB 6MOJ). Model was generated by aligning structures on 188 RBD $\alpha \alpha$ atoms. c-f, Surface representation of RBD epitopes for C135 (blue) (c), S309 (brown, PDB 6WSP) (d), C110 (purple) (e) and REGN-10987 (yellow, PDB 6XDG) (f). Given the low resolution of the antibody-RBD interface, epitopes were assigned by b

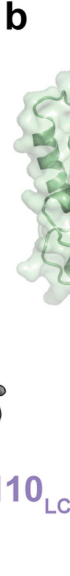

e

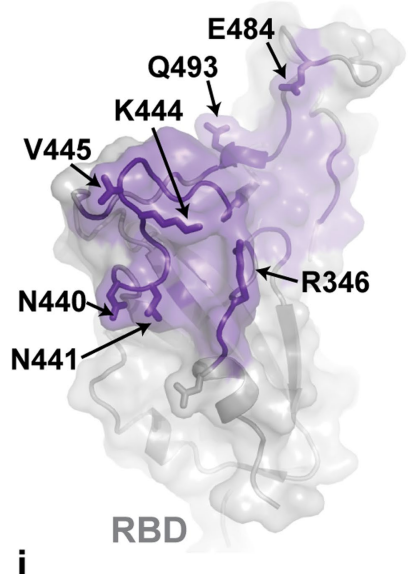

j
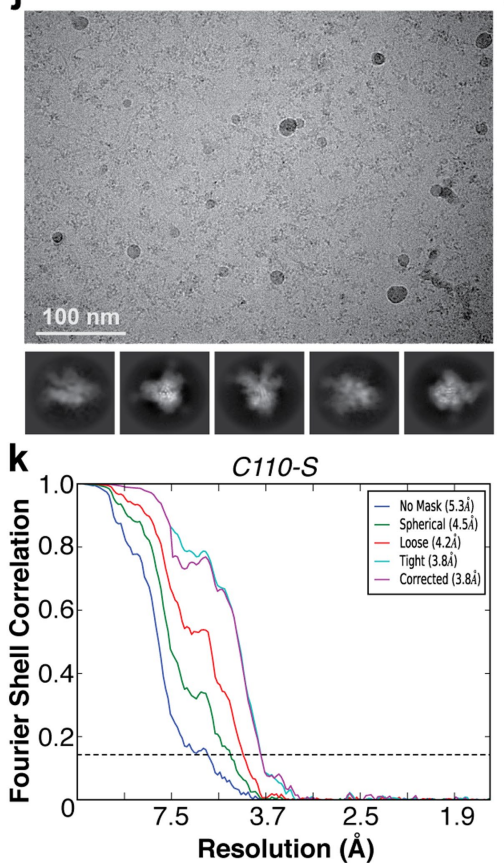

selection of any RBD residue within 7 Å of any antibody $C \alpha$ atom. Mutation sites found in sequence isolates ${ }^{44}$ (green) and in laboratory selection assays ${ }^{40}$ (red) are shown. Representative micrograph selected from total dataset (Supplementary Table 2), 2D class averages, gold-standard FSC plot, and local resolution estimation for $\mathrm{C} 135-\mathrm{S} 2 \mathrm{P}(\mathbf{g}-\mathbf{i})$ and $\mathrm{C} 110-\mathrm{S} 2 \mathrm{P}(\mathbf{j}-\mathbf{I})$. Scale bars, $100 \mathrm{~nm}$. Both complexes revealed binding of Fabs to both two-down and one-up RBD conformations. 


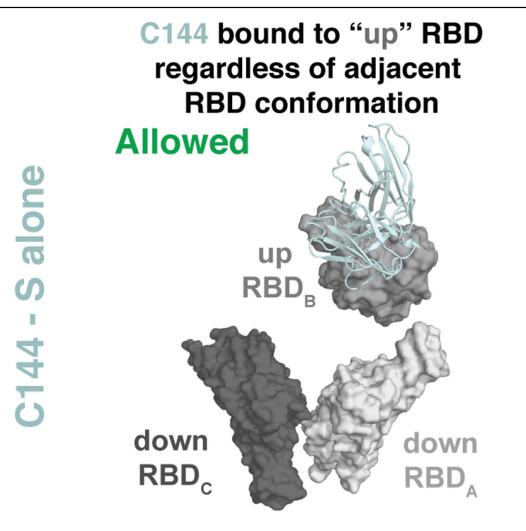

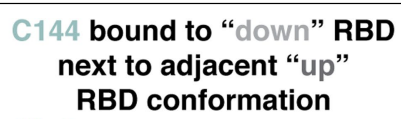

C144 bound to "down" RBD next to adjacent "up" RBD conformation Not

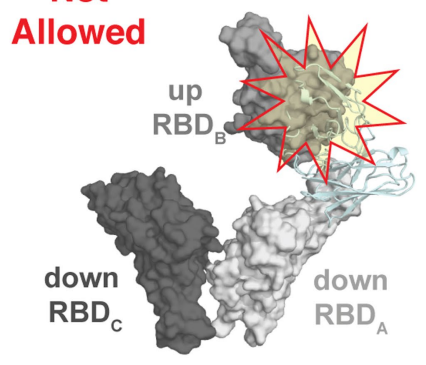

C144 bound to "down" RBD next to adjacent "down" RBD conformation

Allowed

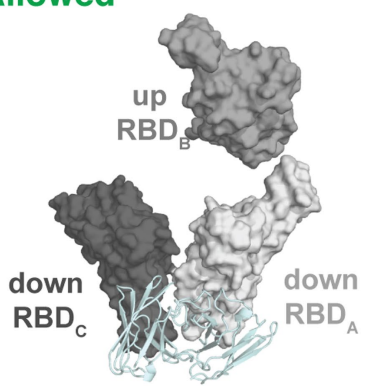

\section{C135 bound to "up" RBD regardless of adjacent RBD conformation \\ C135 bound to "down" RBD regardless of adjacent RBD conformation}

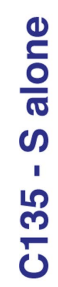

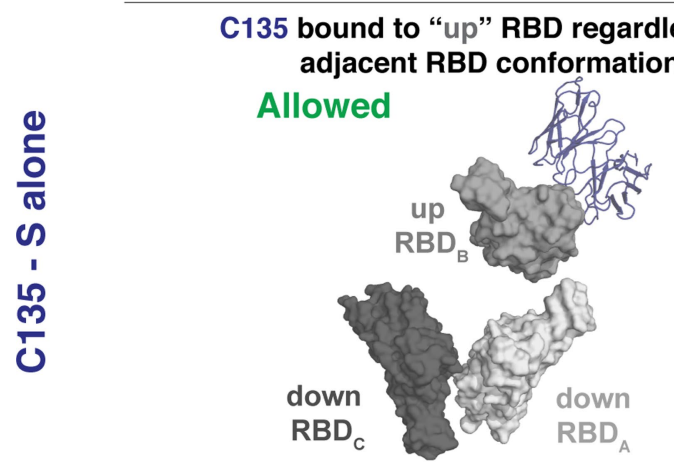

C144 and C135 bind to same "up" RBD, "up" to "down" RBD conformational change creates $\mathrm{C} 144$ quaternary epitope with adjacent "down" RBD

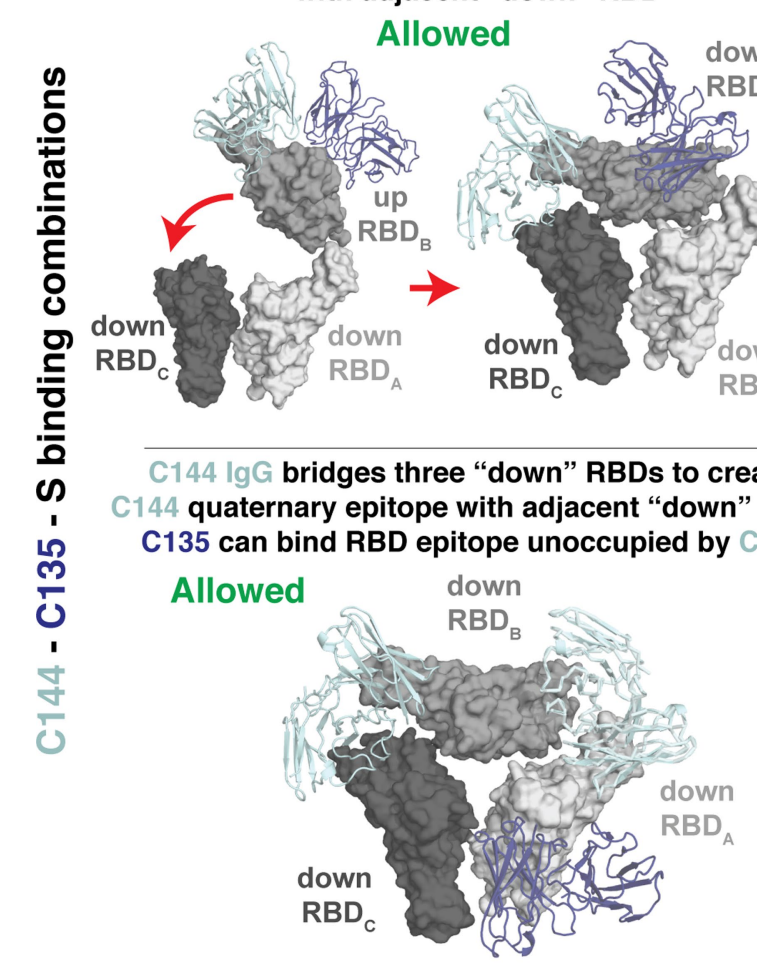

Allowed

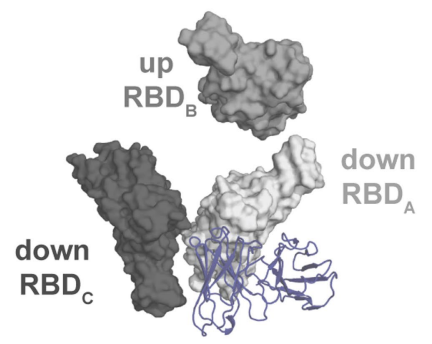

C144 bound to "up" RBD, C135 bound to adjacent "down" RBD, "up" to "dlown" RBD conformational change creates $\mathrm{C} 144$ quaternary epitope with opposite "down" RBD

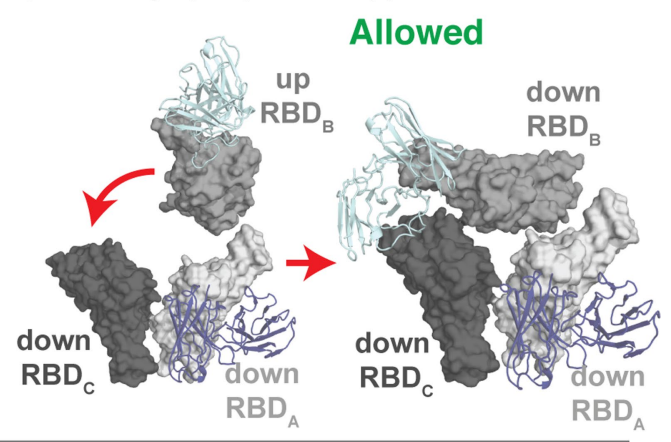

C135 binding with two arms (from different IgGs) on "down" RBDs blocks C144 Ig G bivalent binding

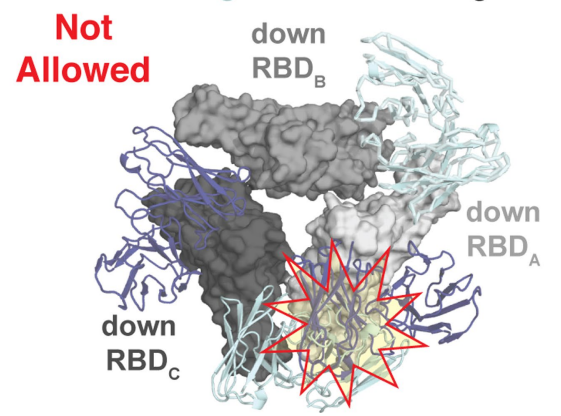

Extended Data Fig. 7 |Possibilities for simultaneous engagement of C144 and C135 on spikes with different combinations of up and down RBDs. Modelling of C144 (light blue) and C135 (dark blue) $\mathrm{VH}_{\mathrm{H}} \mathrm{VL}$ domains on different RBD conformations. Steric clashes are shown as a red and yellow star. 
C102

VH3-53/VK3-20 Class 1

\begin{tabular}{|c|c|c|c|}
\hline RBD & $\begin{array}{l}k_{\mathrm{a}}\left(10^{5}\right) \\
\left(M^{-1} s^{-1}\right)\end{array}$ & $\begin{array}{c}k_{d}\left(10^{-3}\right) \\
\left(s^{-1}\right)\end{array}$ & $K_{\mathrm{D}}(\mathrm{nM})$ \\
\hline wt & 1.4 & 3.8 & 27 \\
\hline R346S & 1.2 & 8.6 & 72 \\
\hline N439K & 1.0 & 3.2 & 32 \\
\hline N440K & 1.4 & 7.6 & 55 \\
\hline A475V & 0.8 & 31.9 & 395 \\
\hline V483A & 1.0 & 3.1 & 32 \\
\hline E484K & 1.4 & 8.7 & 61 \\
\hline $2493 R$ & 2.2 & 17.5 & 81 \\
\hline
\end{tabular}

b

\begin{tabular}{|c|c|c|c|}
\hline RBD & $\begin{array}{l}k_{\mathrm{a}}\left(10^{5}\right) \\
\left(\mathrm{M}^{-1} \mathbf{s}^{-1}\right)\end{array}$ & $\begin{array}{c}k_{d}\left(10^{-3}\right) \\
\left(s^{-1}\right)\end{array}$ & $K_{\mathrm{D}}(\mathrm{nM})$ \\
\hline wt & 2.3 & 4.1 & 18 \\
\hline R346S & 1.1 & 5.6 & 52 \\
\hline N439K & 1.2 & 3.6 & 29 \\
\hline N440K & 1.4 & 5.8 & 40 \\
\hline A475 & 2.3 & 53 & 228 \\
\hline V483A & 1.3 & 4.1 & 32 \\
\hline $\mathrm{E} 484 \mathrm{~K}$ & n.b. & n. & n. \\
\hline Q493R & n.b. & n.b. & n.b. \\
\hline
\end{tabular}

\section{C121}

\begin{tabular}{|c|c|c|c|}
\hline RBD & $\begin{array}{l}k_{\mathrm{a}}\left(10^{5}\right) \\
\left(\mathrm{M}^{-1} \mathrm{~s}^{-1}\right)\end{array}$ & $\begin{array}{c}k_{d}\left(10^{-3}\right) \\
\left(s^{-1}\right)\end{array}$ & $K_{\mathrm{D}}(\mathrm{nM})$ \\
\hline wt & 5.2 & 2.5 & 0.5 \\
\hline R346S & 6.3 & 5 & 0.8 \\
\hline N439K & 5.0 & 3.8 & 0.8 \\
\hline N440K & 6.9 & 4.1 & 0.6 \\
\hline A475V & 6.4 & 4.7 & 0.7 \\
\hline V483A & 2.0 & 1.5 & 0.8 \\
\hline Ë404 & n.b. & n. & n.b. \\
\hline Q493R & 10 & 115 & 111 \\
\hline
\end{tabular}

C

\begin{tabular}{|c|c|c|c|}
\hline \multicolumn{4}{|c|}{$\begin{array}{r}\text { C135 } \\
V H 3-30 / V K 1-5\end{array}$} \\
\hline RBD & $\begin{array}{l}k_{\mathrm{a}}\left(10^{5}\right) \\
\left(M^{-1} s^{-1}\right)\end{array}$ & $\begin{array}{c}k_{\mathrm{d}}\left(10^{-3}\right) \\
\left(\mathrm{s}^{-1}\right)\end{array}$ & $K_{\mathrm{D}}(\mathrm{nM})$ \\
\hline wt & 1.9 & 1.2 & 6 \\
\hline R346S & n.b. & n.b. & n.b. \\
\hline N439K & 1.3 & 4.9 & 37 \\
\hline $\mathrm{N} 440 \mathrm{~K}$ & n. & n. & n. \\
\hline A475V & 1.5 & 1.8 & 12 \\
\hline V483A & 1.4 & 1.3 & 9 \\
\hline E484K & 2.7 & 1.8 & 7 \\
\hline Q493R & 1.3 & 1.7 & 13 \\
\hline
\end{tabular}

C105

VH3-53/VL2-8 Class 1

$k_{\mathrm{a}}\left(10^{5}\right) k_{\mathrm{d}}\left(10^{-3}\right)$

\begin{tabular}{|c|c|c|c|}
\hline RBD & $\left(M^{-1} s^{-1}\right)$ & $\left(s^{-1}\right)$ & $K_{\mathrm{D}}(\mathrm{nM})$ \\
\hline wt & 0.7 & 0.9 & 14 \\
\hline R346S & 0.5 & 1.1 & 20 \\
\hline N439K & 0.6 & 1.1 & 19 \\
\hline N440K & 0.6 & 1.1 & 18 \\
\hline $\mathrm{A} 475 \mathrm{~V}$ & 1.0 & 22 & 225 \\
\hline V483A & 0.5 & 0.8 & 15 \\
\hline E484r & 0.6 & 1.1 & 19 \\
\hline Q493F & 0.7 & 0.4 & 6 \\
\hline
\end{tabular}

C002

VH3-30/VK1-39 Class 2

$k_{\mathrm{a}}\left(10^{5}\right) k_{\mathrm{d}}\left(10^{-3}\right)$

$\operatorname{RBD}\left(\begin{array}{ccc}\left(\mathrm{M}^{-1} \mathbf{s}^{-1}\right) & \left(\mathrm{s}^{-1}\right) & K_{\mathrm{D}}(\mathrm{nM})\end{array}\right.$

\begin{tabular}{r|rrr} 
wt & 8.3 & 9.0 & 11 \\
R346S & 3.2 & 8.6 & 27 \\
N439K & 6.0 & 9.7 & 16 \\
N440K & 3.3 & 8.1 & 24 \\
A475V & 2.1 & 6.3 & 31 \\
V483A & 2.7 & 2.9 & 11 \\
\hline $484 K$ & $n . b$ & $n . b$ & $n . b$ \\
\hline$Q 493 R$ & 1.8 & 106 & 596 \\
\hline
\end{tabular}

\section{C119}

VH1-46/VL2-14 Class 2 $k_{\mathrm{a}}\left(10^{5}\right) k_{\mathrm{d}}\left(10^{-3}\right)$

RBD $\left(\begin{array}{lll}\left(M^{-1} s^{-1}\right) & \left(s^{-1}\right) & K_{\mathrm{D}}(n M)\end{array}\right.$

\begin{tabular}{|c|c|c|c|}
\hline wt & 26 & 26 & 10 \\
\hline R346S & 2.6 & 5.7 & 22 \\
\hline $\mathrm{N} 439 \mathrm{~K}$ & 6.4 & 12.5 & 20 \\
\hline N440K & 1.8 & 3.8 & 21 \\
\hline $\mathrm{A} 475 \mathrm{~V}$ & 1.4 & 2.8 & 20 \\
\hline V483A & 1.7 & 3.7 & 22 \\
\hline${ }^{\star} \mathrm{E} 484 \mathrm{~K}$ & $\begin{array}{l:ll}1.1 & 005\end{array}$ & $\begin{array}{l:l}28 & 2.7\end{array}$ & 91 \\
\hline Q493R & 5.3 & 11 & 20 \\
\hline
\end{tabular}

C110

VH5-51/NK1-5 Class 3 $k_{\mathrm{a}}\left(10^{5}\right) k_{\mathrm{d}}\left(10^{-3}\right)$

$\operatorname{RBD}\left(\mathrm{M}^{-1} \mathbf{s}^{-1}\right) \quad\left(\mathrm{s}^{-1}\right) \quad K_{\mathrm{D}}(\mathrm{nM})$

\begin{tabular}{r|ccc}
\hline wt & 0.7 & 0.09 & 1.3 \\
\hline $\mathrm{N} 346 \mathrm{~S}$ & 0.2 & 1.1 & 68 \\
$\mathrm{~N} 440 \mathrm{~K}$ & 0.4 & 0.3 & 8 \\
$\mathrm{~A} 475 \mathrm{~V}$ & 0.5 & 0.1 & 2 \\
$\mathrm{~V} 483 \mathrm{~A}$ & 0.4 & 0.1 & 3 \\
& 0.09 & 2 \\
\hline $\mathrm{E} 484 \mathrm{~K}$ & 0.4 & 0.9 & 27 \\
\hline
\end{tabular}

$\mathrm{C}_{102}{ }_{\mathrm{HC}}$

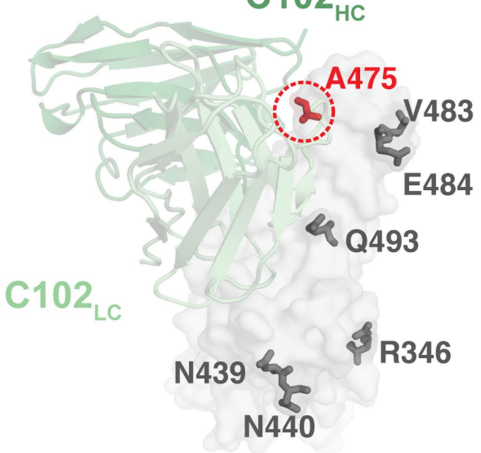

$\mathrm{COO2}_{\mathrm{HC}}$

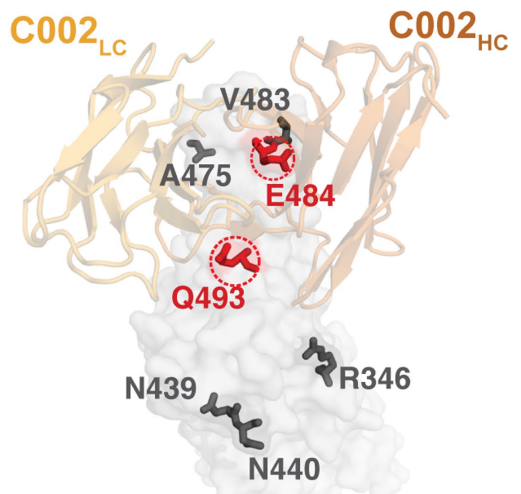

*C104

VH4-34/VK3-20 Class 2

\begin{tabular}{|c|c|c|c|c|}
\hline & & & $\begin{array}{r}k_{d}(1 \\
\left(s^{-1}\right.\end{array}$ & $K_{\mathrm{D}}$ \\
\hline & 1. & & 17.6 & 19 \\
\hline & & & 1 & 9 \\
\hline & & & & \\
\hline & 1. & & 31.2 & 10 \\
\hline & 1. & & 25.0 & 12 \\
\hline & 0.9 & & 24.5 & 69 \\
\hline & n.b. & & n.b. n.b & n.b. \\
\hline 403 & & 0 & \begin{tabular}{l|l}
36.1 & 1
\end{tabular} & 17 \\
\hline
\end{tabular}

Extended Data Fig. 8 | SPR binding data for NAbs. a-c, Kinetic and equilibrium constants for binding to unaltered RBD (indicated as wild type, wt) and mutant RBDs are shown in tables beside structures of a representative NAb-RBD complex for each class. Residues that were mutated are highlighted as coloured side chains on a grey RBD surface. Antibody $\mathrm{VH}-\mathrm{VL}$ domains are shown as cartoons. Kinetic and equilibrium constants for NAbs that contact adjacent RBDs on the S trimer (C144, C002, C119 and C121) do not account for contacts to a secondary RBD because binding was assayed by injected monomeric RBDs over immobilized IgGs. Asterisk indicates kinetic constants determined from a two-state binding model. 
a

Class 1

C102

C105

CC12.1

CC12.3

COVA2-4

B38
CB6

CB6
CB6

REGN10933
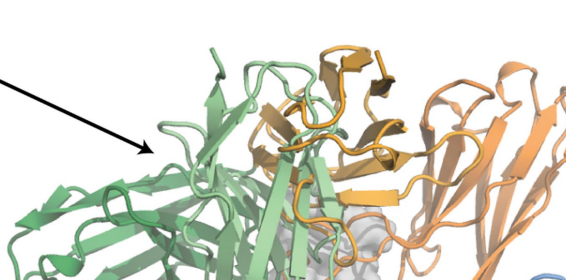

Class 2

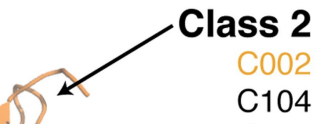

C1002

C119

C121

C144

P2B-2F6

BD23

Ab2-4

5 A 6

COVA2-39*

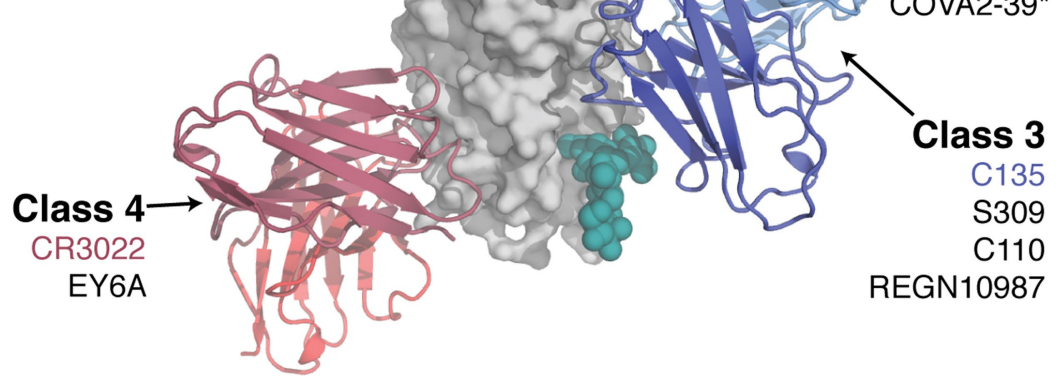

C135

S309

C110 b

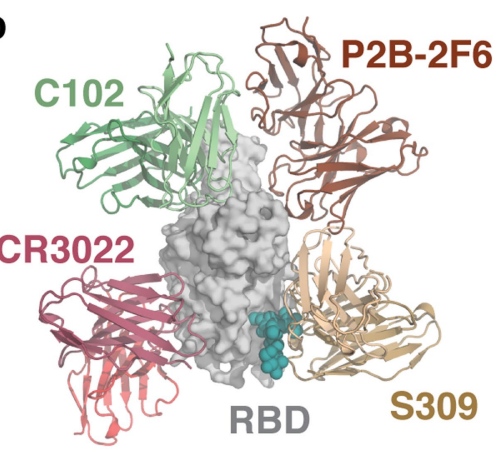

C

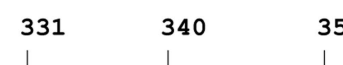

350

360

370

380

।

SARS2-RBD NITNUCPEGEVENATREASUYAWNRKRISHCVADYSVLY

C102

$\mathrm{CC} 12.3$

B38

$\mathrm{C} 002$

C104

C119

C121

P2B-2F6

BD23

C144

COVA2-39

C135

S309

REGN10987

CR3022

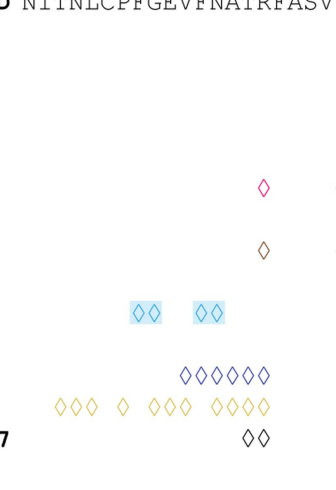

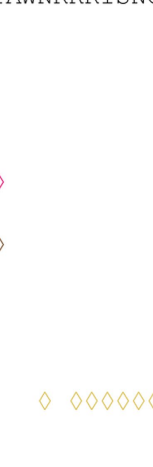

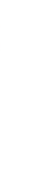

1

390

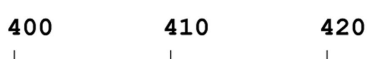

I 100 । DYK

\begin{tabular}{|c|c|c|}
\hline$\diamond$ & & $\diamond \Delta$ \\
\hline$\Delta<$ & $\Delta \Delta$ & 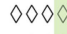 \\
\hline$\diamond \Delta 0$ & $\Delta \diamond$ & $\Delta \Delta \Delta$ \\
\hline
\end{tabular}

$\begin{array}{llllllll}440 & 450 & 460 & 470 & 480 & 490 & 500 & 510\end{array}$

SARS2-RBD LPDDFTGCVIAWNSNNLDSKVGGNYNYLYRLFRKSNLKPFERDISTEIYQAGSTPCNGVEGFNCYFPLQSYGFOPTNGVGYQPYRVVVLSFELL

C102

CC12.3

B38

$\mathrm{C} 002$

C104

C119

C121

P2B-2F 6

BD23

C144

COVA2-39

C135

S309

REGN10987

CR3022

Extended Data Fig. 9 |Summary of NAbs. a, Structural depiction of a representative NAb from each class binding its RBD epitope. b, Composite model illustrating non-overlapping epitopes of NAbs from each class bound to

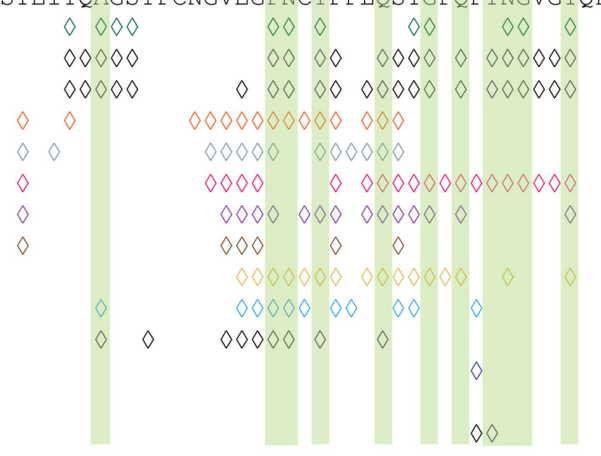

$\Delta \diamond \diamond$

a RBD monomer. c, Epitopes for SARS-CoV-2 NAbs. RBD residues involved in ACE2 binding are boxed in green. Diamonds represent $R B D$ residues contacted by the indicated antibody. 
Extended Data Table 1 | Classification and structural properties of SARS-CoV-2 RBD-specific antibodies

\begin{tabular}{|c|c|c|c|c|c|c|c|c|c|}
\hline Antibody & Reference & $\begin{array}{l}\text { IGHV } \\
\text { (\# of aa } \\
\text { SHM) }\end{array}$ & $\begin{array}{l}\text { CDRH3 } \\
\text { length } \\
(\mathrm{aa})^{\wedge}\end{array}$ & $\begin{array}{l}\text { IGLV } \\
\text { (\# of aa } \\
\text { SHM) }\end{array}$ & $\begin{array}{l}\text { CDRL3 } \\
\text { length } \\
(\mathrm{aa})^{\wedge}\end{array}$ & $\begin{array}{l}\mathrm{IC}_{50} / \mathrm{IC}_{90} \\
(\mathrm{ng} / \mathrm{mL}) \dagger\end{array}$ & $\begin{array}{l}\text { Potential } \\
\text { IgG intra- } \\
\text { spike } \\
\text { binding }^{\S}\end{array}$ & $\begin{array}{l}\text { Contacts } \\
\text { adjacent } \\
\text { RBD }\end{array}$ & Structural Information \\
\hline \multicolumn{10}{|c|}{ Class 1: Blocks ACE2, accessibility of RBD epitope only in "up" conformation } \\
\hline $\begin{array}{c}\text { C102 } \\
\end{array}$ & this study & VH3-53 (2) & 11 & VK3-20 (0) & 9 & $34 / 143$ & ??? & $? ? ?$ & 3.0 A Fab-RBD \\
\hline C105 & Barnes, et al. ${ }^{26}$ & VH3-53 (0) & 12 & $V L 2-8(1)$ & 11 & $26.1 / 134$ & Yes & No & $3.4 \AA$ Fab-S. PDB 6 XCM \\
\hline B38 & $\mathrm{Wu}$, et al. ${ }^{28}$ & $\mathrm{VH} 3-53(1)$ & 9 & VK1-9 (2) & 10 & $117 / \mathrm{NA}$ & ??? & $? ? ?$ & $1.8 \AA$ Fab-RBD, PDB $7 B Z 5$ \\
\hline CC12.3 & Yuan, et al..$^{30}$ & VH3-53 (3) & 12 & VK3-20 (1) & 9 & $20 / N A$ & $? ? ?$ & $? ? ?$ & 2.9 Å. Fab-RBD, PDB 6XC7 \\
\hline \multicolumn{10}{|c|}{ Class 2: Blocks ACE2, accessibility of RBD epitope in "up"/"down" conformations } \\
\hline $\mathrm{C} 002$ & this study & VH3-30 (1) & 17 & $V K 1-39(1)$ & 9 & $8.9 / 37.6$ & Yes & Yes & $3.4 \AA$ Fab-S \\
\hline C104 & this study & VH4-34 (6) & 17 & $V K 3-20(3)$ & 9 & $23.3 / 140$ & Yes & Yes & $3.7 \AA \mathrm{Fab}-\mathrm{S}$ \\
\hline C119 & this study & VH1-46 (1) & 20 & VL2-14 (3) & 11 & $9.1 / 97.8$ & Yes & Yes & $3.5 \AA \mathrm{Fab}-\mathrm{S}$ \\
\hline C121 & this study & VH1-2 (2) & 22 & VL2-23 (0) & 10 & $6.7 / 22.3$ & Yes & Yes & $3.6 \AA \mathrm{Fab}-\mathrm{S}$ \\
\hline C144 & this study & VH3-53 (3) & 25 & VL2-14 (1) & 10 & $6.9 / 29.7$ & Yes & Yes & $3.3 \AA$ Fab-S \\
\hline COVA2-39 & Wu, et al. ${ }^{38}$ & VH3-53 (3) & 17 & VL2-23 (1) & 10 & 36 / NA & $? ? ?$ & $? ? ?$ & 1.7 Å Fab-RBD, PDB 7JMP \\
\hline $5 \mathrm{~A} 6$ & Wang, et al. ${ }^{41}$ & & & & & $75.5 / \mathrm{NA}$ & Yes & Yes & $2.4 \AA \mathrm{Fab}-\mathrm{S}$ \\
\hline P2B-2F6 & $\mathrm{Ju}$, et al. ${ }^{27}$ & VH4-38*02 (2) & 20 & $V L 2-8(0)$ & 10 & $50 / N A$ & $? ? ?$ & $? ? ?$ & $2.9 \AA$ Fab-RBD, PDB 7BWJ \\
\hline $\mathrm{Ab} 2-4$ & Liu, et al. ${ }^{10}$ & VH1-2 (3) & 15 & $V L 2-8(0)$ & 10 & 394 / NA & Yes & No & 3.2 A Fab-S, PDB 6 XEY \\
\hline BD23 & Cao, et al. ${ }^{13}$ & $V H 7-4^{*} 02(0)$ & 19 & $V K 1-5^{\star} 03(0)$ & 9 & $4800 / \mathrm{NA}$ & No & No & $3.8 \AA$ Fab-S, PDB 7BYR \\
\hline \multicolumn{10}{|c|}{ Class 3: Does not overlap with ACE2 binding site, accessibility of RBD epitope in "up"/"down" conformations } \\
\hline C135 & this study & VH3-30 (4) & 12 & VK1-5 (3) & 9 & $16.6 / 48.9$ & No & No & $3.5 \AA$ Fab-S \\
\hline S309 & Pinto, et al. ${ }^{34}$ & VH1-18 (6) & 20 & $V K 3-20(3)$ & 8 & $79^{*} / \mathrm{NA}$ & No & No & 3.1 A Fab-S, PDB 6WPS \\
\hline C110 & this study & VH5-51 (2) & 21 & $V K 1-5(3)$ & 9 & $18.4 / 77.3$ & No & No & $3.8 \AA \mathrm{Fab}-\mathrm{S}$ \\
\hline REGN10987 & Hansen, et al. $^{6}$ & VH3-30 (4) & 13 & VL2-14 (6) & 10 & $6.1 / \mathrm{NA}$ & $? ? ?$ & $? ? ?$ & 3.9 Å Fab-RBD, PDB 6XDG \\
\hline \multicolumn{10}{|c|}{ Class 4: Does not overlap with ACE2 binding site, accessibility of RBD epitope only in "up" conformation } \\
\hline CR3022 & Yuan, et al. $^{9}$ & VH5-51 (8) & 12 & VK4-1 (3) & 9 & $>10,000 / \mathrm{NA}$ & ??? & $? ? ?$ & 3.1 Å Fab-RBD, PDB 6W41 \\
\hline Cov1-16 & Liu, et al. ${ }^{68}$ & VH1-46 (1) & 20 & $V K 1-33(3)$ & 10 & $130 / \mathrm{NA}$ & $? ? ?$ & $? ? ?$ & $2.9 \AA \mathrm{Fab}-\mathrm{RBD}$ \\
\hline EY6A & Zhou, et al. ${ }^{69}$ & $V H 3-30 * 18(3)$ & 14 & $V K 1-39(0)$ & 10 & $70-20,000^{* *} / \mathrm{NA}$ & No & Yes & 3.7 A Fab-S, PDB 6ZDH \\
\hline S304 & Pinto, et al. ${ }^{34}$ & VH3-13 (5) & 14 & $V K 1-39(6)$ & 10 & $>5,000 / \mathrm{NA}$ & & & 4.3 Å Fab-S, PDB 7JW0 \\
\hline $\mathrm{S} 2 \mathrm{~A} 4$ & Piccoli, et al. ${ }^{70}$ & VH3-7 (2) & $?$ & $V L 2-23(0)$ & $?$ & $3,500 / \mathrm{NA}$ & No & Yes & 3.3 A Fab-S, PDB 7JVC \\
\hline
\end{tabular}

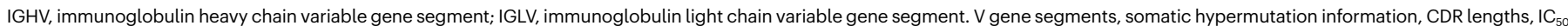
and $I_{90}$ values for NAbs in this study are from ref. ${ }^{32}$. The following references are cited in the table: refs. ${ }^{6,9,10,13,26-28,30,34,38,41,67-69}$.

${ }^{\wedge}$ Average human antibody CDRH3 and CDRL3 lengths are 15 (CDRH3) and 9-10 (CDRL3) amino acids.

${ }^{*} \mathrm{C}_{50}$ value calculated against authentic SARS-CoV-2 virus.

${ }^{* *} \mid \mathrm{C}_{50}$ values varied depending on neutralization assay used.

+Unknown $\mathrm{IC}_{90}$ values indicated as NA (not available).

§Potential for intra-spike crosslinking by an lgG binding to a single spike trimer was evaluated as described in the Methods.

?Sequence information not available.

???Inference that cannot be made from a structure of a Fab bound to a RBD. 


\section{Reporting Summary}

Nature Research wishes to improve the reproducibility of the work that we publish. This form provides structure for consistency and transparency in reporting. For further information on Nature Research policies, see our Editorial Policies and the Editorial Policy Checklist.

\section{Statistics}

For all statistical analyses, confirm that the following items are present in the figure legend, table legend, main text, or Methods section.

$\mathrm{n} / \mathrm{a} \mid$ Confirmed

$\square$ The exact sample size $(n)$ for each experimental group/condition, given as a discrete number and unit of measurement

$\square$ A statement on whether measurements were taken from distinct samples or whether the same sample was measured repeatedly

$\triangle$ The statistical test(s) used AND whether they are one- or two-sided

$\triangle$ Only common tests should be described solely by name; describe more complex techniques in the Methods section.

$\bigotimes \square$ A description of all covariates tested

Х $\square$ A description of any assumptions or corrections, such as tests of normality and adjustment for multiple comparisons

$\triangle$ A full description of the statistical parameters including central tendency (e.g. means) or other basic estimates (e.g. regression coefficient)

AND variation (e.g. standard deviation) or associated estimates of uncertainty (e.g. confidence intervals)

$\triangle \square$ For null hypothesis testing, the test statistic (e.g. $F, t, r$ ) with confidence intervals, effect sizes, degrees of freedom and $P$ value noted $\bigotimes$ Give P values as exact values whenever suitable.

$\bigotimes \square$ For Bayesian analysis, information on the choice of priors and Markov chain Monte Carlo settings

$\triangle \square$ For hierarchical and complex designs, identification of the appropriate level for tests and full reporting of outcomes

\ $\square$ Estimates of effect sizes (e.g. Cohen's $d$, Pearson's $r$ ), indicating how they were calculated

Our web collection on statistics for biologists contains articles on many of the points above.

\section{Software and code}

Policy information about availability of computer code

Data collection SerialEM automated image acquisition software version 3.7; BLU-ICE X-ray data collection software at SSRL; Tecan Infinite M1000 plate reader software; Biacore T200 instrument software

Data analysis GraphPad Prism 8.4.3 was used for polyreactivity data analysis; XDS (Build 20200417), Xia2 v0.3.8, DIALS v2.2, and the CCP4 v7.0.6 suite of crystallographic programs (AIMLESS v0.7.4, PHASER v2.8.2), Sculptor v2.0, Phenix v1.18, Coot v0.8.9, cryoSPARC v2.15, CTFFIND4 v4.1.14, PyMOL v2.2, Chimera v1.13, ChimeraX-v1.0, and Molprobity v4.4 were used for structural analysis; Kinetic constants were calculated using Biacore T200 Evaluation Software v3.2; PDBePISA v1.48 for BSA calculations;

For manuscripts utilizing custom algorithms or software that are central to the research but not yet described in published literature, software must be made available to editors and reviewers. We strongly encourage code deposition in a community repository (e.g. GitHub). See the Nature Research guidelines for submitting code \& software for further information.

\section{Data}

Policy information about availability of data

All manuscripts must include a data availability statement. This statement should provide the following information, where applicable:

- Accession codes, unique identifiers, or web links for publicly available datasets

- A list of figures that have associated raw data

- A description of any restrictions on data availability

The atomic models generated from X-ray crystallographic studies of the C102-RBD complex, C102 Fab, C002 Fab, C110 Fab, C121 Fab, and C135 Fab have been deposited at the Protein Data Bank (PDB, http://www.rcsb.org/) under accession codes PDB 7K8M, 7K8N, 7K80, 7K8P, 7K8Q, and 7K8R, respectively. The atomic models and cryo-EM maps generated from cryo-EM studies of the C002-S 2P (state 1), C002-S 2P (state 2), C104-S 2P, C110-S 2P, C119-S 2P, C121-S 2P (state 1), C121-S 2P (state 2), C135-S 2P and C144-S 6P complexes have been deposited at the PDB (http://www.rcsb.org/) and the Electron Microscopy Databank (EMDB, 


\section{Field-specific reporting}

Please select the one below that is the best fit for your research. If you are not sure, read the appropriate sections before making your selection.
Х Life sciences
$\square$ Behavioural \& social sciences
Ecological, evolutionary \& environmental sciences

For a reference copy of the document with all sections, see nature.com/documents/nr-reporting-summary-flat.pdf

\section{Life sciences study design}

All studies must disclose on these points even when the disclosure is negative.

Sample size Not applicable to this study, because no sample size calculation was performed to design the study.

Data exclusions No data were excluded.

Replication All experiments were replicated successfully, $\mathrm{n}$ values can be found in figure legends.

Randomization Not applicable to this study, as we do not report experiments that use randomized data.

Blinding Not applicable to this study, as we do not report any experiments that applied blinding.

\section{Reporting for specific materials, systems and methods}

We require information from authors about some types of materials, experimental systems and methods used in many studies. Here, indicate whether each material, system or method listed is relevant to your study. If you are not sure if a list item applies to your research, read the appropriate section before selecting a response.

\begin{tabular}{l|l} 
Materials \& experimental system \\
\hline n/a Involved in the study \\
$\square$ & $\square$ Antibodies \\
$\square$ & $\square$ Eukaryotic cell lines \\
$\square$ & $\square$ Animals and other organisms \\
$\square$ & $\square$ Clinical data \\
\hline & $\square$ Dual use research of concern
\end{tabular}

\section{Antibodies}

Antibodies used

Validation
HRP-conjugated anti-human IgG secondary antibody (SouthernBiotech, Catalog 2040-04; Goat Anti-Human IgG-HRP; Lot B3919.NEB80B) used at a 1:5000 dilution. All other antibodies reported were expressed in this study.

Primary antibodies reported in this study were described previously in Robbiani et al. (doi:10.1038/s41586-020-2456-9). Target validation was done with multiple binding assays and structural studies using cryo-EM and X-ray crystallography. Control anti-HIV-1 antibodies reported in this study have been previously described and validated in a polyreactivity assay (Schoofs, et al. doi:10.1016/ j.immuni.2019.04.014). Reactivity of the primary antibody listed above is based on the information on manufacturer's homepages.

\section{Eukaryotic cell lines}

Policy information about cell lines

Cell line source(s)

Authentication

Mycoplasma contamination

Commonly misidentified lines (See ICLAC register)
Expi293F (ThermoFisher cat. A14527)

Not authenticated after purchase.

The cell lines were not contaminated by mycoplasma as determined by using the Lonza Mycoplasma Detection Kit.

No commonly misidentified cell lines were used. 